\title{
Occurrence of species of the genus Pityophthorus Eichhoff (Coleoptera, Curculionidae, Scolytinae) in the province of Quebec, Canada
}

\author{
Valentin Popa', Louis Morneau², Céline Piché2, \\ André Deshaies ${ }^{3}$, Eric Bauce ${ }^{4}$, Claude Guertin'
}

I INRS-Institut Armand-Frappier, 531 des Prairies Boulevard, Laval, Quebec, Canada, H7V 1 B7 2 Ministère des Ressources naturelles du Québec, DPF, 2700 Einstein Street, Québec, Quebec, Canada, G1P 3 W8 3 Ministère des Ressources naturelles du Québec, DGPSP, 880 Chemin Sainte-Foy, Québec, Quebec, Canada, G1S 4 X4 4 Université Laval, DBF, 2320 Des Bibliothèques Street, Québec, Quebec, Canada, G1V OA6

Corresponding author: Claude Guertin (claude.guertin@iaf.inrs.ca)

Academic editor:M.Alonso-Zarazaga | Received 1 August 2013|Accepted 11 October 2013| Published 12 November 2013

Citation: Popa V, Morneau L, Piché C, Deshaies A, Bauce E, Guertin C (2013) Occurrence of species of the genus Pityophthorus Eichhoff (Coleoptera, Curculionidae, Scolytinae) in the province of Quebec, Canada. ZooKeys 348: $97-$ 124. doi: $10.3897 /$ zookeys.348.6029

\begin{abstract}
Twig beetles in the genus Pityophthorus Eichhoff, 1864 include more than 300 species worldwide, with maximum diversity in tropical and subtropical regions. To date, approximately 50 species of Pityophthorus have been recorded in Canada, and these species are associated mainly with coniferous trees. Since 1981, no comprehensive study on this difficult taxonomic group has been conducted in Quebec, Canada, most likely due to their limited significance as forest pests. Based on data gathered from five years of field sampling in conifer seed orchards and compiled from various entomological collections, the distribution of Pityophthorus species in Quebec is presented. Approximately 291 new localities were recorded for the Pityophthorus species. Five species-group taxa, namely P. puberulus (LeConte, 1868), P. pulchellus pulchellus Eichhoff, 1869, P. pulicarius (Zimmermann, 1868), P. nitidus Swaine, 1917, and P. cariniceps LeConte\&Horn, 1876 were the most widespread. In contrast, P. consimilis LeConte, 1878, P. intextus Swaine, 1917, P. dentifrons Blackman, 1922, P. ramiperda Swaine, 1917, and P. concavus Blackman, 1928 display a notably limited distribution. In addition, the first distribution records of $P$. intextus and $P$. biovalis Blackman, 1922 are furnished, and the subspecies P. murrayanae murrayanae Blackman, 1922 is reported from Quebec for the second time. Moreover, distribution maps are provided for all Pityophthorus species recorded in the province of Quebec.
\end{abstract}

Copyright Valentin Popa et al. This is an open access article distributed under the terms of the Creative Commons Attribution License 3.0 (CC-BY), which permits unrestricted use, distribution, and reproduction in any medium, provided the original author and source are credited. 


\section{Keywords}

Distribution, fauna, locality, Quebec, records, twig beetle

\section{Introduction}

Twig beetles in the genus Pityophthorus Eichhoff, 1864 include approximately 386 species distributed worldwide (Bright 1981; Bright and Skidmore 1997, 2002; Wood and Bright 1992) with 218 species found in North and Central America (Bright 1981; Wood 1982), and approximately 55 other species found in South America (Wood 2007). More than $70 \%$ of the Pityophthorus world fauna is distributed in North, Central, and South America. Wood (2007) notes that the genus Pityophthorus has an American origin and displays its maximum diversity in subtropical and tropical areas. A vast majority of the North and Central American species of this genus breed in twigs of coniferous trees (Bright 1981; Wood 1982), whereas the South American species breed predominantly in deciduous trees (Wood 2007).

Excluding the taxonomic works, twig beetles have received little attention from the scientific community and forest managers simply because these organisms generally colonize declining trees or tree parts. They are often found in thin-barked parts of stressed or weakened trees, and they are rarely associated with extensive epidemics in forest ecosystems (Furniss and Carolin 1977). Nevertheless, some reports have indicated that several Pityophthorus species may become problematic and cause medium to severe damage in coniferous plantations (Rappaport and Wood 1994; Stevens et al. 1979). Furthermore, twig beetles may vector pathogenic fungi. For example, pitch canker disease of Monterey pine, Pinus radiata D. Don, is caused by wounding and transmission of a fungal pathogen to twigs during feeding by a complex of Pityophthorus sp. in California (Dallara 1997; Hoover et al. 1996; Sakamoto et al. 2007). In a Monterey pine plantation in Spain, approximately $25 \%$ of the analyzed population of Pityophthorus pubescens (Marsham) was responsible for carrying the spores of Fusarium circinatum Nirenberg and O'Donnell, which is the fungus that causes pitch canker disease (Romón et al. 2007). More recently, Kolařik et al. (2011) reported that another Pityophthorus species, the walnut twig beetle, $P$. juglandis Blackman, is an important vector of the fungus Geosmithia morbida (M. Kolařik, E. Freeland, C. Utley and Tisserat 2011). This complex causes thousand cankers disease, which is a serious necrosis of the phloem of walnut trees, Juglans sp., in the United States (Seybold et al. 2013). The walnut twig beetle has expanded its range considerably in the U.S. (Cranshaw 2011; Seybold et al. 2012), which may explain the recent attention that the disease has attracted from the forest management community (Seybold et al. 2013).

The species of the genus Pityophthorus are difficult to identify with morphological techniques. Both sexes are often required for accurate identification at the species level (Bright 1981; Wood 1982, 2007). Taxonomic revisions of this genus in North America have been published by LeConte and Horn (1876), Swaine (1918), and Blackman (1928). Wood (1978) placed the genus Pityophthorus into the Corthylini and the 
Pityophthorina (subtribe). The Pityophthorus are closely related to Araptus Eichhoff, 1872 whose species are found mainly in Mexico, Central, and South America (Wood 2007; Wood and Bright 1987, 1992). A major contribution to the knowledge of the Pityophthorus was provided by Bright (1981), who published a taxonomic monograph in which more than 220 species from Central and North America were described and classified.

In Canada, approximately 50 species of Pityophthorus have been recorded (Bright 1968; Bright 1971, 1976, 1981; Bright and Skidmore 1991, 1997, 2002; Bright et al. 1994; Wood and Bright 1987, 1992). In the province of Quebec, Canada, 17 species have been reported (Bright 1976, 1981; Bright and Skidmore 1997, 2002; Laplante et al. 1991; McNamara 1991; MRNQ 2008). However, no comprehensive and upto-date study has been performed on the genus Pityophthorus in Quebec since the publications of D.E. Bright.

The purpose of this article is to update the distribution of all recorded Pityophthorus species in the province of Quebec, Canada. The species records are based on data collected from several entomological collections and on our own field-trapping data obtained during the last five years (2008-2012). A study of the distribution of Pityophthorus species is highly important, particularly in ecological and biological studies on different species and for further taxonomic revisions of this difficult bark beetle group. This study also provides background information for researchers working with exotic forest insects and for forest managers.

\section{Methods and conventions}

This article is based on data obtained from Pityophthorus specimens collected exclusively in the province of Quebec, Canada. Two different sources of data were used: field captures performed between 2008 and 2012 and a survey of public and private entomological collections.

Field collection methods. The field data included in this article were collected over a period of five years (2008-2012) from trapping activities conducted in six different seed orchards scattered from west to east over diverse types of landscapes in the province of Quebec (Table 1). These seed orchards are composed mainly of white pine, Pinus strobus L., but also contain specimens of red pine, Pinus resinosa Ait. and jack pine, Pinus banksiana Lamb., as well as white spruce, Picea glauca (Moench) Voss, red spruce, Picea rubens Sargent, and black spruce, Picea mariana Miller, Briton, Sterns \& Poggenburg.

Two types of trapping techniques were used in the study sites. The first trapping technique employed "Yellow Japanese Beetle" (YJB) traps (Trécé Inc., Adair, Oklahoma, United States) equipped with 500-ml Mason ${ }^{\circledast}$ jars. Each jar was filled with 50 $\mathrm{ml}$ of propylene glycol to kill and preserve the trapped insects. The YJB traps were baited with a polyethylene "bubble cap" release device (Contech Enterprises Inc., Delta, British Columbia, Canada) that contained ( \pm ) trans-pityol (release rate of $0.2 \mathrm{mg} /$ day). Pityol is an aggregation pheromone component of several Pityophthorus species 
Table I. The sampled seed orchards with their corresponding area in hectares, their geographic coordinates and the nearest locality.

\begin{tabular}{cccc}
\hline Seed orchard & Nearest locality & Geographic coordinates (decimal degrees) & Area (ha) \\
\hline Huddersfield & Fort-Coulonge & $45.9215 ;-76.6219$ & 9.7 \\
Dorion & Lac Cayamant & $46.0459 ;-76.2828$ & 6.6 \\
Verchères & Saint-Amable & $45.6773 ;-73.3303$ & 5.5 \\
Cleveland & Saint-Claude & $45.6764 ;-71.9954$ & 2.5 \\
Aubin-de-l'Isle & Saint-Simon-les-Mines & $46.2089 ;-70.6780$ & 4.5 \\
Cap-Tourmente & Cap-Tourmente & $47.0680 ;-70.8076$ & 2.8 \\
\hline
\end{tabular}

(Brauner and De Groot 2007; Dallara et al. 2000; De Groot and De Barr 2000; Francke et al. 1987; López et al. 2011). The population monitoring of Pityophthorus species in the six seed orchards was performed starting in 2008 and ending in 2012. The biological material was collected with an average frequency of twice a month from mid-April to the end of September.

A second trapping technique was used to increase the chance of capture of other Pityophthorus specimens that may not respond to synthetic pityol alone as an attractant. Twelve-unit Lindgren funnel traps were used to monitor Pityophthorus populations in three of the six previously mentioned seed orchards, namely Verchères, Huddersfield and Cleveland (Table 1). The Lindgren traps were placed at a density of approximately three traps per hectare and were baited with $( \pm)$ trans-pityol and UHR ethanol (200 $\mathrm{mg}$ /day, Synergy Semiochemicals Corp., Burnaby, British Columbia). Ethanol is an attractant for large number of bark and ambrosia beetles (Miller and Rabaglia 2009). Lindgren traps were equipped with plastic trap cups filled with $50 \mathrm{ml}$ of propylene glycol. The cups were emptied at the same frequency as described in the previous trapping technique. The field-collected Pityophthorus specimens were preserved in $70 \%$ ethanol and subsequently mounted and pinned. All of the captured specimens originating from field trapping are preserved in the INRS-Institute Armand-Frappier (INRS-IAF) entomological collection. The species identification was performed according to morphological criteria by using a Discovery V-20 stereomicroscope (Carl Zeiss Canada Ltd.) equipped with an ICc3 video camera. Pictures of the identified and unidentified Pityophthorus species are available at the following web address: www. profs.inrs.ca/cguertin/ZOOKEYS_2013/MENU.html

The field data were complemented with information gathered from six public and two private entomological collections. In this article, the following acronyms are used for the public entomological collections:

CNC Canadian National Collection of Insects, Arachnids and Nematodes, Agriculture and Agri-Food Canada, Ottawa, ON, Canada;

MRNQ Ministère des Ressources Naturelles du Québec, Québec, QC, Canada;

LFRC Natural Resources Canada, Laurentian Forestry Research Center, RenéMartineau Insectarium, Québec, QC, Canada; 
ECLU Entomological Collection, Laval University, Québec, QC, Canada;

LEMU Lyman Entomological Collection, McGill University, Montreal, Quebec, Canada;

ROUM Robert-Ouellet Entomological Collection, Montreal University, Montreal, QC, Canada.

The acronyms used for the private entomological collections are the following:

CCC Claude Chantal's Collection, Varennes, QC, Canada

CLC Claire Lévesque’s Collection, Sherbrooke, QC, Canada

The following two additional acronyms are also employed in this article:

SLWC S.L. Wood entomological collection. Although this collection was not directly examined, some $P$. lautus Eichhoff specimens captured in the province of Quebec are deposited there. Even though the specimens have not been examined, these are included in the article as reliable records that were published by Bright (1981).

INRS-IAF Entomological collection of the INRS-Institut Armand-Frappier, Laval, QC, Canada. All specimens collected during the field trapping activities are deposited in this collection.

Approximately $50 \%$ of the specimens analyzed in this study belong to MRNQ entomological collection. In general, to capture bark beetle specimens, the "Ministère des Ressources naturelles du Québec" (MRNQ) uses permanent sampling stations dispersed in all representative types of forest ecosystems and landscapes across the province of Quebec.

Other conventions and symbols. The distribution record for each of the Pityophthorus species mentioned in this article is displayed using the following sequences: 1 . Name of the locality where the specimen was captured. In some cases, instead of the name of the locality, the historic territorial administrative name may appear (e.g., "Township"), because no locality name has been assigned to the sampling area. 2. Name of the Regional County Municipality (RCM) to which the cited locality belongs. Regional County Municipalities have delineated the province of Quebec territory since 1979. Many localities in Quebec that are situated in different geographic areas have received the same name. To avoid any confusion relative to the locality names, the corresponding RCM is included. 3. Date of capture. If the capture date is missing or it is unreadable on the label, a question mark is included. 4. Number of examined specimens and the acronym of the entomological collection to which they belong. These data are included within parentheses and separated by a coma. 5. Name of host tree species, displayed in italics. In some cases, the host tree data are missing.

The five abovementioned sequences are separated by comas. Locality records are separated by semicolons (;). If a species was recorded many times in the same locality, 
the various dates of capture are separated by a slash symbol ( / ). For each mentioned species, the distribution data are presented in a manner that previous literature records are separated from the new records to highlight the originality of the article. The general distribution of each species in Canada is presented at the end of each species record. The following abbreviations were used for the provinces and territories: YT-Yukon Territory, NT-Northwest Territories, NU-Nunavut, BC-British Columbia, AB-Alberta, SK-Saskatchewan, MB-Manitoba, ON-Ontario, QC-Quebec, NB-New Brunswick, PE-Prince Edward Island, NS-Nova Scotia, and NF \& LB-Newfoundland and Labrador. The distribution records of all Pityophthorus species in Canada are presented according to Bright (1981), McNamara (1991), Wood and Bright (1992), and Bright and Skidmore (1997). The distribution of each Pityophthorus species was mapped by using the ARCGIS and ARCMAP software starting from an EXCEL database, which is available at the following web link: www.profs.inrs.ca/cguertin/ZOOKEYS_2013/MENU.html

\section{Results}

To date, the following 17 Pityophthorus species have been recorded in the province of Quebec, Canada:

\section{Pityophthorus lautus Eichhoff, 1872}

http://species-id.net/wiki/Pityophthorus_lautus

Fig. 7

Records from Bright (1981). Aylmer, Communauté-Urbaine-de-l'Outaouais, 14VIII-1920, (3, CNC); Wakefield, Les Collines-de-l'Outaouais, 11-V-1951, (19, SLWC), Rhus typhina; Sainte-Anne-de-Bellevue, Montréal, ?, (10, CNC) / 2 individuals who supposedly originated from the same samples were found at LEMU and display an unreadable label.

New records. Montréal, 14-V-1936, (9, ECLU); Mont Saint-Hilaire, La-Valléedu-Richelieu, ?, (1, LFRC), Mont Saint-Bruno, La-Vallée-du-Richelieu, ?, (1, LFRC). Distribution in Canada. NT, ON, QC, NB, NS.

Pityophthorus pulicarius (Zimmermann, 1868) http://species-id.net/wiki/Pityophthorus_pulicarius Fig. 3

Records from Bright (1981). Chelsea, Les Collines-de-l'Outaouais, 20-VI-1917, (6, CNC); Grand-Remous, La-Vallée-de-la-Gatineau, 17-VIII-1978, (2, LFRC), Pinus banksiana; Wychwood, Communauté-Urbaine-de-l'Outaouais, 21-VI-1917, (2, CNC); Pointe à David, La-Vallée-de-la-Gatineau, 2-VI-1975, (1, LFRC), Pi- 


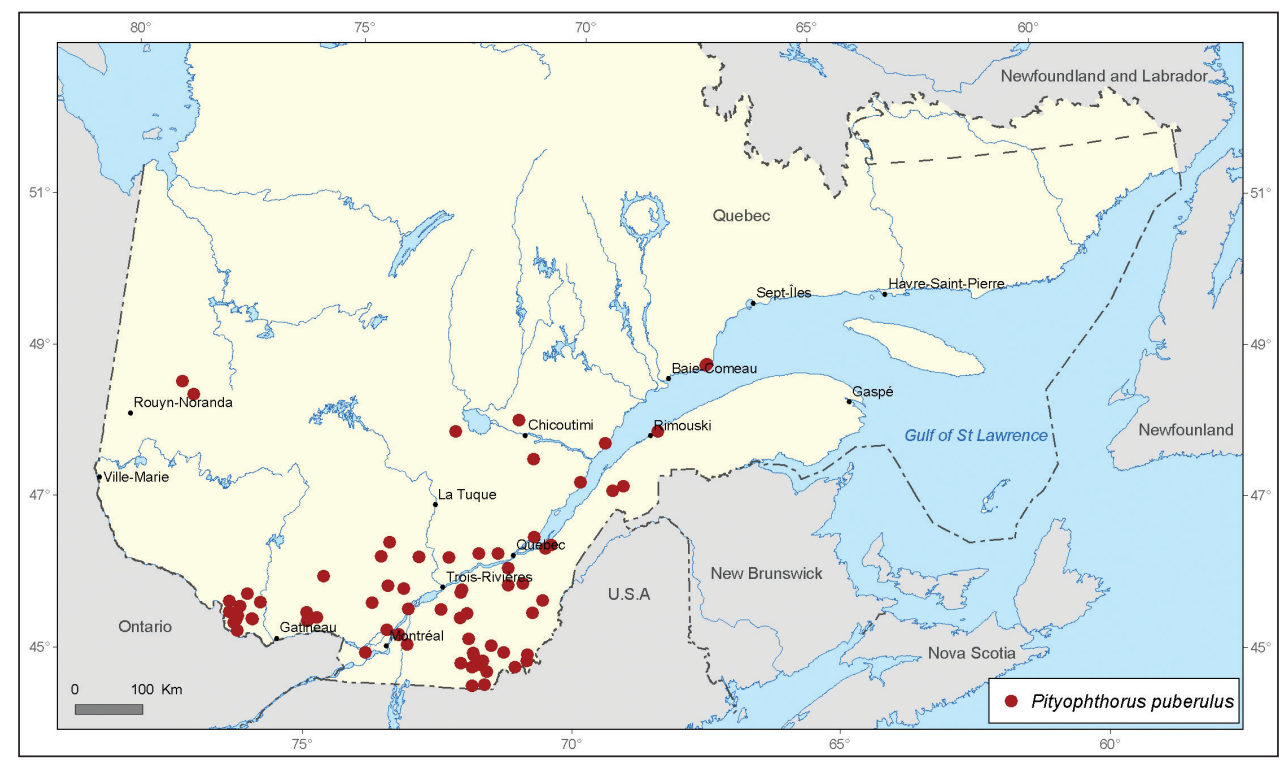

Figure I. Map of Pityophthorus puberulus (LeConte) records in Quebec, Canada.

nus banksiana; Lac Louvicourt, La-Vallée-de-l'Or, 1-IX-1978, (2, LFRC), Pinus banksiana; Moffet, Témiscamingue, 16-VIII-1978, (2, LFRC), Pinus banksiana; Rivière-aux-Rats, Le Haut-Saint-Maurice, 14-VII-1978, (1, LFRC), Pinus banksiana; Sainte-Anne-de-Bellevue, Montréal, ?, (1, CNC).

New records. Chute-Saint-Philippe, Antoine-Labelle, 22-VIII-1990, (1, MRNQ), Pinus banksiana / 11-IX-1990, (3, MRNQ), Pinus banksiana; Chemin du Lac Petawaga, Antoine-Labelle, 3-VII-1981, (1, MRNQ), Pinus banksiana; L'Annonciation, Antoine-Labelle, 30-VI-1985, (3, MRNQ), Pinus resinosa; Lac Landron, La Vallée-dela-Gatineau, 5-VI-1982, (2, MRNQ), Pinus banksiana; Baie Mercier, La Vallée-de-laGatineau, 16-VIII-1979, (1, MRNQ), Pinus banksiana; Lac Pageot, Pontiac, 12-VII1983, (1, MRNQ), Pinus banksiana; Vinton, Pontiac, 31-V-2011, (1, MRNQ), Pinus resinosa; Chemin du Lac de l'Épine, La Vallée-de-l'Or, 8-VII-1981, (1, MRNQ), Pinus banksiana; Lac Quentin, La Vallée-de-l'Or, 31-VII-1982, (5, MRNQ), Pinus banksiana; Mont Saint-Michel, La-Vallée-de-l'Or, 6-VII-2011, (1, MRNQ), Pinus banksiana; Notre-Dame-du-Nord, Témiscamingue, 16-VII-1985, (2, MRNQ), Pinus resinosa; Guérin, Témiscamingue, 31-VI-1982, (1, MRNQ), Pinus banksiana; Lac Bend, Témiscamingue, 21-VII-1981, (1, MRNQ), Pinus sylvestris; Latulipe, Témiscamingue, 24-VII-1981, (1, MRNQ), Pinus banksiana; Lac Nodier, Témiscamingue, 16-VII-1981, (1, MRNQ), Pinus banksiana; Lac des Seize, Témiscamingue, 13-VII1983, (2, MRNQ), Pinus banksiana; Saint-Bruno-de-Guigues, Témiscamingue, 22VII-2005, (1, MRNQ), Pinus banksiana; Lac à Bédard, Témiscamingue, 22-VII-1986, (1, MRNQ), Pinus banksiana; Cloutier, Rouyn-Noranda, 29-VII-1981, (2, MRNQ); Pinus banksiana; Lac Bruyère, Rouyn-Noranda, 20-VI-1983, (1, MRNQ), Pinus banksiana; Lac Lavoie, Rouyn-Noranda, 15-VII-1981, (1, MRNQ), Pinus banksiana; Lac 
McWatters, Rouyn-Noranda, 17-VII-1984, (2, MRNQ), Pinus banksiana; La Morandière, Abitibi, 13-VII-1984, (2, MRNQ), Pinus banksiana; Villemontel, Abitibi, 6-VII-1983, (4, LFRC), Pinus banksiana; Lac Macamic, Abitibi Ouest, 26-VI-1984, (1, MRNQ), Pinus banksiana; Saint-Dominique, Abitibi Ouest, 14-VII-2011, (1, MRNQ), Pinus banksiana; Saint-Georges, Le Centre-de-la-Mauricie, 22-VII-1981, (3, MRNQ), Pinus resinosa; Saint-Antoine-Abbé, Le Haut-Saint-Laurent, 22-V-2002, (2, MRNQ), Pinus resinosa; Saint-Polycarpe, Vaudreuil-Soulange, 5-VII-2002, (1, MRNQ), Pinus strobus; Lac Wet, Le Haut-Saint-Maurice, 13-VIII-1992, (1, MRNQ), Pinus banksiana; Lefebvre, Drummond, 28-V-1999, (4, MRNQ), Pinus resinosa; Lac Roméo, Jamésie, 20-VI-1981, (1, MRNQ), Pinus banksiana.

Distribution in Canada. SK, MB, ON, QC, NB, NS.

Pityophthorus nitidus Swaine, 1917

http://species-id.net/wiki/Pityophthorus_nitidus

Fig. 4

Records from Bright (1981). Tullochgorum, Le Haut-Saint-Laurent, 20-IX-1910, (2, CNC); Sainte-Anne-de-Bellevue, Montreal, ?, (2, CNC); Saint-Gabriel-de-Rimouski, La Mitis, 8-VII-1970, (6, CCC), Picea glauca.

New records. Les Étroits, Témiscouata, 24-VII-1986, (2, MRNQ), Pinus resinosa; Saint-Éleuthère, Témiscouata, 2-VIII-1984, (11, MRNQ), Picea glauca / 1-VIII-1984, (2, MRNQ), Pinus resinosa / 17-VIII-1989, (3, MRNQ); Estcourt, Témiscouata, 1-VIII-1984, (2, MRNQ), Pinus resinosa; Lac Nadreau, La Côte-de-Beaupré, 9-VIII1989, (16, MRNQ), Picea mariana / 31-VII-1990, (16, MRNQ), Picea mariana; SaintAlphonse-de-Caplan, Bonaventure, 8-IX-1989, (1, MRNQ); Saint-Elzéar, Bonaventure, 27-VI-1984, (5, MRNQ), Pinus sylvestris; Lac du Curé, Bonaventure, 17-VI1996, (4, MRNQ); Rivington, Argenteuil, 1-IX-1992, (10, MRNQ), Pinus banksiana; Kinnear's Mills, Les Appalaches, 26-VIII-1993, (9, MRNQ), Picea glauca / 10-IX1993, (3, MRNQ), Picea glauca; Sacré-Cour-de-Marie, Les Appalaches, 5-VIII-1981, (5, MRNQ), Picea abies; Routhierville, La Matapédia, 31-VII-1996, (8,MRNQ), Picea glauca; Saint-Paul-de-Montminy, Montmagny, 24-VII-1981, (3, MRNQ), Pinus resinosa; Saint-Joseph-de-Ham, Les Sources, 21-VII-1981, (1, MRNQ), Pinus resinosa; Les Éboulements, Charlevoix, 4-VI-1981, (1, MRNQ), Pinus strobus; Saint-Hilarion, Charlevoix, 8-VIII-1990, (1, MRNQ), Pinus strobus; Petite-Rivière-Saint-François, Charlevoix, 19-VIII-1985, (2, MRNQ), Picea abies; Lac Rivard, Maria-Chapdelaine, 20-VII-2011, (1, MRNQ), Picea mariana; Lac des Trois Élans, Maria-Chapdelaine, 1-VIII-2001, (1, MRNQ), Picea mariana; Pointe-Lebel, Manicouagan, 7-VIII-1981, (1, MRNQ), Pinus resinosa; Sainte-Eulalie, Nicolet-Yamaska, 11-IX-1984, (2, MRNQ), Picea abies; Saint-Thomas-de-Cherbourg, Matane, 9-VIII-1979, (2, MRNQ), Pinus banksiana; Ruisseau Ernest, Antoine-Labelle, 6-VI-1981, (4, MRNQ), Pinus strobus; Scotstown, Le Haut-Saint-François, 26-VIII-1981, (3, MRNQ), Picea glauca; SaintFélix-d'Otis, Le Fjord-du-Saguenay, 24-IX-1984, (6, MRNQ), Pinus resinosa; Réser- 


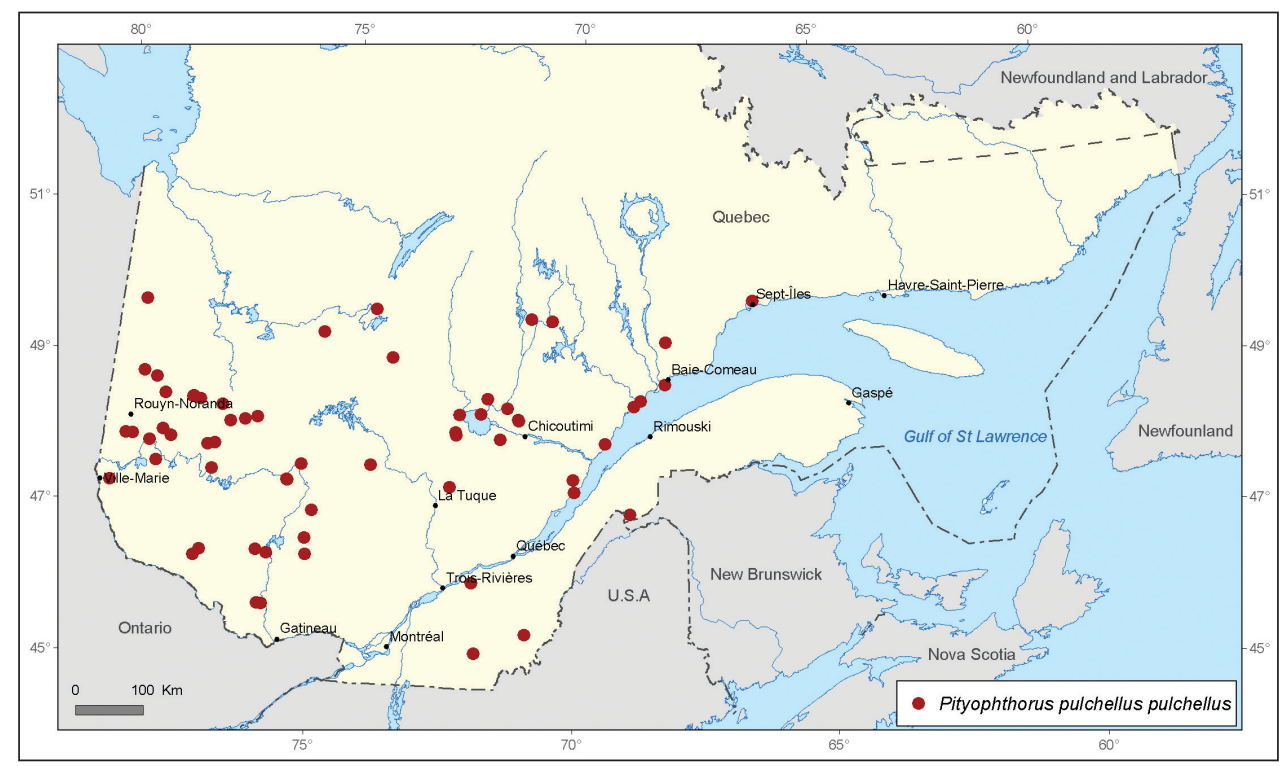

Figure 2. Map of Pityophthorus pulchellus pulchellus Eichhoff records in Quebec, Canada.

voir Baskatong, La Vallée-de-la-Gatineau, 26-VI-1981, (2, MRNQ), Pinus banksiana; Île d'Anticosti, Minganie, 12-VI-1973, (4, CCC); Longue-Pointe-de-Mingan, Minganie, 25-VII-1980, (2, CCC); Sept-Îles, Sept-Rivières, 1-VIII-1982, (2, CCC), Picea mariana / 13-VI-1984, (1, CCC) / 8-VI-1985, (71, CCC) / 27-VII-1985, (1, CCC) / 3-VIII-1985, (30, CCC), Picea mariana / 24-V-1986, (5, CCC) / 6-VII-1986, (2, CCC) / 5-VIII-1986, (1, CCC) / 29-V-1987, (5, CCC) / 1-VI-1987, (1, CCC) / 7-VI-1987, (1, CCC) / 9-VII-1987, (2, CCC) / 16-VI-1988, (5, CCC) / 12-VII-1988, (1, CCC) / 2-VI-1990, (58, CCC) / 26-VII-1990, (1, CCC) / 23-VIII-1990, (16, CCC) / 10-VI1991, (1, CCC) / 17-VII-1991, (11, CCC) / 20-VII-1991, (10, CCC) / 30-VII-1991, (1, CCC) / 6-VIII-1991, (4, CCC) / 9-VIII-1992, (4, CCC).

Distribution in Canada. NT, YT, AB, BC, ON, QC, NB, NS, NF \& LB.

Pityophthorus intextus Swaine, 1917

http://species-id.net/wiki/Pityophthorus_intextus

Fig. 9

New records. Saint-Hilarion, Charlevoix, 8-VIII-1990, (1, MRNQ), Picea glauca; Lac Fourcet, Antoine-Labelle, 9-VII-1981, (1, MRNQ), Picea mariana; Saint-Hérménégilde, Coaticook, 5-VII-1985, (1, MRNQ), Pinus resionsa; Lac Poutrincourt, Le Domaine-du-Roy, 25-VII-2007, (1, MRNQ), Pinus banksiana; Sept-Îles, SeptRivières, 13-VII-1987, (1, CCC) / 16-VI-1988, (1, CCC).

Distribution in Canada. BC, AB, SK, MB, ON, QC, NB, NS, NF \& LB. 
Pityophthorus pulchellus pulchellus Eichhoff, 1869

http://species-id.net/wiki/Pityophthorus_pulchellus_pulchellus

Fig. 2

Records from Bright (1981). Kazabazua, La-Vallée-de-la-Gatineau, 13-XII-1917, (5, CNC), Pinus banksiana; Lac Saint-Jean, Lac Saint-Jean E, ?, (6, LFRC), Pinus banksiana; Sainte-Anne-du-Lac (Zec Mitchinamécus), Antoine-Labelle, 4-VII1978, (48, LFRC), Pinus banksiana.

Records from Paquin and Dupérré (2001). Jamésie, 14-IX-1997, (1, LEMU), Picea mariana.

New records. Sainte-Françoise, Bécancour, 18-VI-1986, (4, MRNQ), Pinus strobus; Colombier (Serres), La Haute-Côte-Nord, 16-VII-1986, (2, MRNQ), Pinus resinosa; Les Escoumins, La Haute-Côte-Nord, 6-VI-1984, (1, CCC); SaintAmbroise, Le Fjord-du-Saguenay, 6-VII-1992, (1, MRNQ), Pinus strobus; Lac du Grand Détour, Le Fjord-du-Saguenay, 20-VII-2011, (1, MRNQ), Pinus banksiana; Saint-David-de-Falardeau, Le Fjord-du-Saguenay, 9-VII-1996, (1, MRNQ), Pinus resinosa; Sept-Îles, Sept-Rivières, 8-VI-1985, (4, CCC) / 16-V-1986, (3, CCC) / 6-VII-1986 (1, CCC) / 29-V-1987, (1, CCC) / 17-VI-1987, (1, CCC) / 25-VI1987, (2, CCC) / 13-VII-1987, (15, CCC) / 12-VI-1988, (3, CCC) / 21-VI-1988, (7, CCC) / 4-VII-1988, (3, CCC) / 19-V-1989, (1, CCC) / 9-VI-1989, (1, CCC) / 27-V-1990, (7, CCC) / 2-VI-1990, (99, CCC) / 26-VII-1990, (1, CCC) / 27-VIII1990, (3, CCC) / 29-V-1991, (22, CCC) / 12-VIII-1991, (1, CCC) / 22-V-1992, (2, CCC); Lac Saint-Ludger, Lac Saint-Jean-Est, 18-VII-1981, (3, MRNQ), Pinus banksiana; Ruisseau du Pont, Lac Saint-Jean-Est, 31-VII-1984, (10, MRNQ), Pinus banksiana; Notre-Dame-du-Rosaire, Lac Saint-Jean-Est, 1-VII-1981, (1, MRNQ), Picea glauca; Lac Fourcet, Antoine-Labelle, 9-VII-1981, (1, MRNQ), Picea mariana; Chute-Saint-Philippe, Antoine-Labelle, 16-VI-1995, (4, MRNQ), Pinus banksiana; Landrienne, Abitibi, 15-VII-1987, (2, MRNQ), Pinus banksiana; Authier Nord, Abitibi Ouest, 13-VII-1979, (1, MRNQ), Pinus banksiana; La Morandière, Abitibi, 11-VII-1984, (9, MRNQ), Pinus banksiana; Guyenne Township, Abitibi, 16-VIII-1983, (1, MRNQ), Pinus banksiana; Lac Castagnier, Abitibi, 9-IX-1986, (3, MRNQ), Pinus banksiana; Villemontel, Abitibi, 31-VIII-1983, (80, LFRC), Pinus banksiana; Lac Dubois, Témiscamingue, 21-VIII-1986, (1, MRNQ), Pinus banksiana; Lorainville, Témiscamigue, 4-VI-1981, (51, MRNQ), Pinus banksiana; Lac Nodier, Témiscamingue, 4-VIII-1981, (1, MRNQ), Pinus banksiana; Cloutier, Rouyn-Noranda, 30-VII-1981, (4, MRNQ), Pinus banksiana; Lac Surimau, Rouyn-Noranda, 11-VIII-1982, (5, MRNQ), Pinus banksiana; RapideDeux, Rouyn-Noranda, 6-VI-1983, (1, MRNQ), Pinus banksiana; Lac Charles, Le Haut-Saint-Maurice , 17-VII-1987, (1, MRNQ), Pinus banksiana; Lac Wet, Le Haut-Saint-Maurice, 13-VIII-1992, (15, MRNQ), Pinus banksiana; Lac Gosselin, Le Haut-Saint-Maurice, 1-VIII-1981, (6, MRNQ), Pinus banksiana; Lac Louvicourt, La-Vallée-de-l'Or, 6-IX-1984, (1, MRNQ), Pinus banksiana; Lac Tremblay, La-Vallée-de-l'Or, 27-VII-2011, (1, MRNQ), Pinus banksiana; Rivière Mégis- 


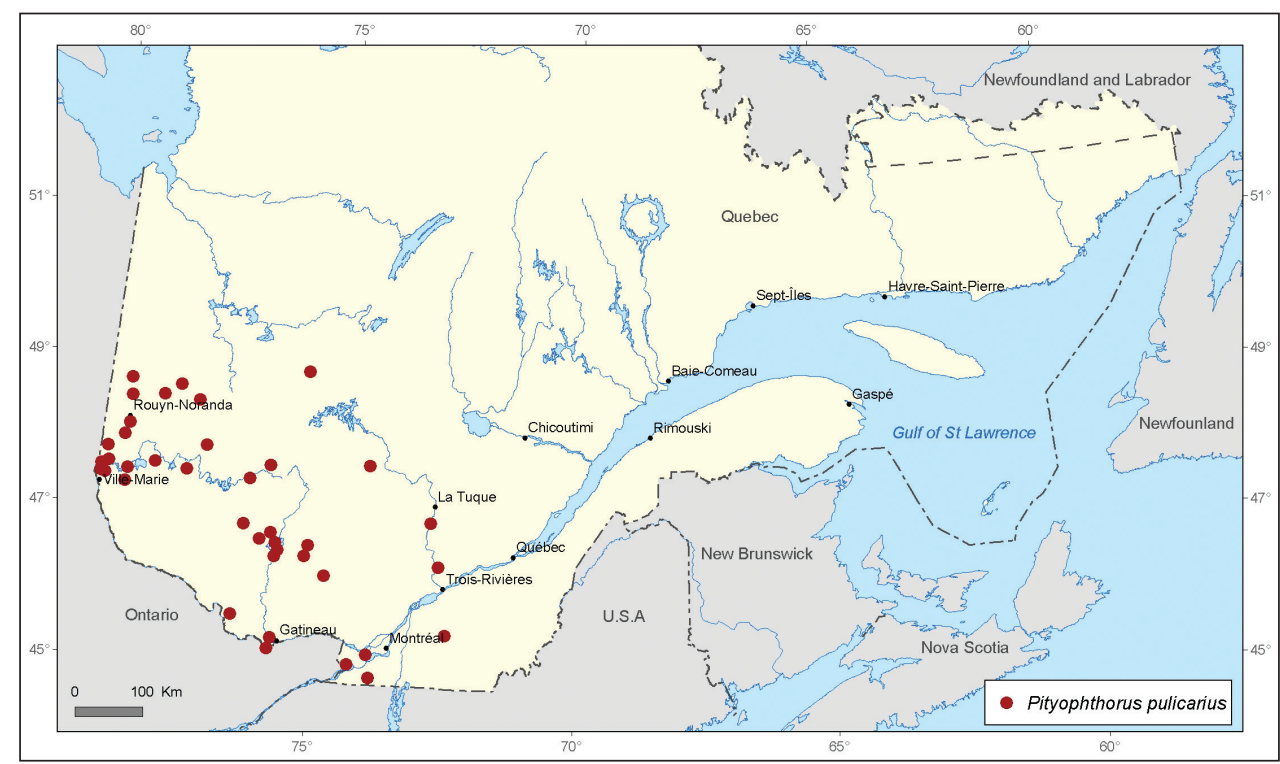

Figure 3. Map of Pityophthorus pulicarius (Zimmermann) records in Quebec, Canada.

cane, La-Vallée-de-l'Or, 12-VI-1981, (5, MRNQ), Pinus banksiana; Lac Faillon, La-Vallée-de-l'Or, 16-VI-1981, (2, MRNQ), Pinus banksiana; Lac Villebon, LaVallée-de-l'Or, 6-VI-1981, (4, MRNQ), Pinus banksiana / 2-IX-1981, (4, MRNQ), Pinus banksiana; Lac Fournière, La-Vallée-de-l'Or, 4-VIII-1981, (3, MRNQ), Pinus banksiana / 7-VIII-1981, (3, MRNQ), Pinus banksiana; Lac Prospère, La-Valléede-l'Or, 22-VIII-1981, (3, MRNQ), Pinus banksiana; Lac Palouse, La-Vallée-del'Or, 9-VI-1983, (26, MRNQ), Pinus banksiana; Pointe-Lebel Airport, Manicouagan, 17-VIII-1981, (2, MRNQ), Pinus resinosa; Lac Pistuacanis, Manicouagan, 18-VII-1981, (4, MRNQ), Pinus banksiana; Rivière Betsiamites, Manicouagan, 5-VIII-1981, (1, MRNQ), Pinus banksiana; Lac Saint-Pierre, Le Domaine-du-Roy, 17-VI-1981, (4, MRNQ), Pinus banksiana; Saint-Félicien, Le Domaine-du-Roy, 26-V-2006, (5, MRNQ), Pinus banksiana; Lac Beemer, Le Domaine-du-Roy, 18VII-2007, (2, MRNQ), Pinus banksiana; Lac Mignault, Le Domaine-du-Roy, 15IX-2005, (1, MRNQ), Picea sp.; Rivière Désert, La Vallée-de-la-Gatineau, 12-VII1984, (1, MRNQ), Pinus strobus; Lac Rond, La Vallée-de-la-Gatineau, 22-VI-1983, (52, MRNQ), Pinus banksiana; Lac des Outaouais, La Vallée-de-la-Gatineau, 18VIII-1981, (6, MRNQ), Pinus banksiana; Lac Mosher, La Vallée-de-la-Gatineau, 26-VII-1983, (9, MRNQ), Pinus banksiana; Lac Danford, La Vallée-de-la-Gatineau, 16-VIII-1980, (3, MRNQ), Pinus banksiana / 13-IX-1983, (3, MRNQ), Pinus resinosa; Kazabazua, La Vallée-de-la-Gatineau, 13-XII-1971, (4, MRNQ), Pinus resinosa I 28-V-1984 (7, MRNQ), Pinus resinosa; Lac Dickson, Pontiac, 16-VII-1992, (1, MRNQ), Pinus banksiana; Lac Nigault, Pontiac, 29-VIII-1994, (2, MRNQ), Pinus resinosa; Lac Charrette, Pontiac, 5-VIII-1981, (2, MRNQ), Pinus strobus; Bas-del'Anse, Charlevoix-Est, 18-VI-1981, (2, MRNQ), Pinus sylvestris; Lac Port au Sau- 
mon, Charlevoix-Est, 23-VII-1981, (2, MRNQ), Pinus banksiana; Saint-Sébastiende-Frontenac, Le Granit, 16-VII-1981, (4, MRNQ), Pinus banksiana; Lac Rivaille, Maria-Chapdelaine, 28-VIII-2008, (6, MRNQ), Pinus banksiana; Bromptonville, Sherbrooke, 31-VII-1981, (1, MRNQ), Pinus sylvestris; Lac Winsch, Jamésie, 12VIII-1981, (1, MRNQ), Pinus banksiana; Les Étroits, Témiscouata, 24-VII-1986, (3, MRNQ), Pinus resinosa.

Distribution in Canada. YT, NT, BC, AB, SK, MB, ON, QC, NB.

Pityophthorus cariniceps LeConte \& Horn, 1876 http://species-id.net/wiki/Pityophthorus_cariniceps Fig. 5

Records from Bright (1981). Sainte-Anne-de-Bellevue, Montréal, 17-VIII-1910, (1, CNC), Pinus sp.; L'Île Perrot, Vaudreuil-Soulanges, various dates, (42, CNC) / 4 specimens supposedly originating from the same samples were found at LEMU, Pinus sp.; Old Chelsea, Les Collines-de-l'Outaouais, 23-VI-1966, (14, CNC), Pinus strobus; Wychwood, Communauté-Urbaine-de-l'Outaouais, 2-VI-1917, (5, CNC), Pinus resinosa.

New records. Ways-Mills, Coaticoock, 13-V-1988, (1, MRNQ), Pinus strobus; Beebe Plain, Memphrémagog, 5-VI-1989, (1, MRNQ), Pinus strobus; Saint-Aimédes-Lacs, Charlevoix-Est, 26-VI-1992, (4, MRNQ), Pinus strobus; Shawville, Pontiac, 9-V-2001, (3, MRNQ), Pinus resinosa; Lac Hickey, Pontiac, 14-V-2007, (2, MRNQ), Pinus strobus; Lac Prendergast, Pontiac, 25-V-1981, (1, MRNQ), Pinus strobus; Thorne Centre, Pontiac, 26-V-2003, (2, MRNQ), Pinus resinosa; Baie du Chat, Pontiac, 5-VII-1981, (5, MRNQ), Pinus strobus; Fort Coulonge, Pontiac, various dates in the period 2008-2012, (124, INRS-IAF), Pinus strobus; Lac Cayamant, Pontiac, various dates in the period 2008-2012, (22, INRS-IAF), Pinus strobus; Lac Ruthledge, Les Collines-de-l'Outaouais, 18-VII-1981, (2, MRNQ), Pinus strobus; Sainte-Cécile-de-Masham, Les Collines-de-l'Outaouais, 31-VII-1972, (10, LFRC), Pinus sylvestris; Saint-Charles-de-Mandeville, D'Autray, 13-VII-1981, (5, MRNQ), Pinus strobus; Saint-Zéphirin, Nicolet-Yamaska, 29-VI-1981, (4, MRNQ), Pinus strobus; Les Éboulements, Charlevoix, 4-VI-1981, (2, MRNQ), Pinus strobus; Camp l'Oasis, Portneuf, 12-VI-1980, (1, MRNQ), Pinus strobus; Island Brook, Les HautSaint-François, 30-VII-1981, (1, MRNQ), Pinus resinosa; Cookshire, Le HautSaint-François, 26-IV-2002, (2, MRNQ), Pinus banksiana; Lemieux, Bécancour, 27-VII-1981, (2, MRNQ), Pinus banksiana; Zec Chauvin, La Haute-Côte-Nord, 13-IV-1983, (5, MRNQ), Pinus strobus / 8-VI-1983, (2, MRNQ), Pinus strobus; Mont-Saint-Hilaire, La-Vallée-du-Richelieu, 24-V-1916, (1, LEMU), Pinus strobus; Oka, Deux-Montagnes, 5-VII-1978, (1, CCC), Pinus strobus; Saint-Amable, Marguerite-D’Youville, 11-V-1999, (3, MRNQ), Pinus strobus / various dates in the period 2008-2012, (1051, INRS-IAF), Pinus strobus; Saint-Claude, Le-Val-SaintFrançois, various dates in the period 2008-2012, (85, INRS-IAF), Pinus strobus; 


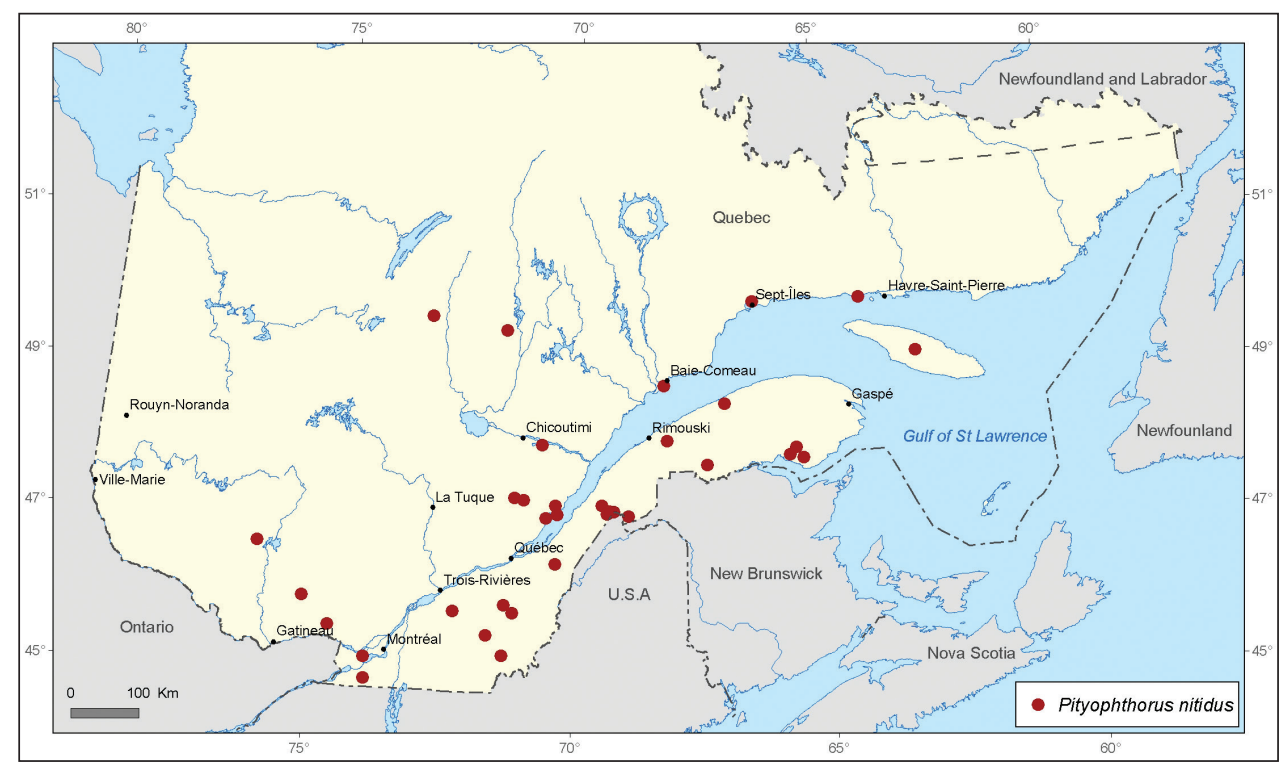

Figure 4. Map of Pityophthorus nitidus Swaine records in Quebec, Canada.

Saint-Simon-les-Mines, Beauce-Sartigan, various dates in the period 2008-2012, (93, INRS-IAF), Pinus strobus; Cap-Tourmente, La Côte-de-Beaupré, various dates in the period 2008-2012, (178, INRS-IAF), Pinus strobus; Sept-Îles, Sept-Rivières, 8-VI-1985, (1, CCC) / 12-VII-1986, (1, CCC) / 13-VI-1987, (1, CCC) / 10-VII1987, (1, CCC) / 18-VI-1988, (1, CCC) / 21-VI-1988, (1, CCC) / 4-VII-1988, (2, CCC) / 27-V-1990, (1, CCC) / 29-V-1991, (3, CCC) / 29-VII-1991, (1, CCC); Lac Ramsay, Les Collines-de-l'Outaouais, 18-V-2009, (1, CCC); Johnville, Compton, 13-V-1987 (1, CLC) / 17-V-1987 (2, CLC) / 20-V-1987 (1, CLC) / 22-V-1988 (1, CLC) / 17-V-1989 (3, CLC) / 21-V-1989 (1, CLC) / 22-X-1989 (1, CLC).

Distribution in Canada. AB, SK, MB, ON, QC, NB, NS.

Pityophthorus biovalis Blackman, 1922

http://species-id.net/wiki/Pityophthorus_biovalis

Fig. 6

New records. L'Annonciation, Antoine-Labelle, 20-VII-1992, (4, MRNQ), Pinus resinosa; Saint-Philémon, Bellechasse, 29-V-1981, (4, MRNQ), Pinus banksiana I 20-VII-1981, (5, MRNQ), Picea abies; Lac du Port au Saumon, Charlevoix-Est, 23-VII-1981, (3, MRNQ), Pinus banksiana; Saint-Claude, Le Val-Saint-François, 20-VII-1992, (5, MRNQ), Picea glauca / 28-VII-2008, (2, INRS-IAF), Pinus strobus / 9-VI-2011, (5, INRS-IAF), Pinus strobus; Quatre-Chemins, Les Pays-d'enHaut, 18-V-1993, (4, MRNQ), Pinus strobus; Woburn, Le Granit, 9-VII-1981, (2, MRNQ), Pinus resinosa; Lac Cayamant, La-Vallée-de-la-Gatineau, 15-VII-2009, (1, 
INRS-IAF), Dosquet, Lotbinière, 20-V-1972, (1, CCC); Saguenay, Le Fjord-duSaguenay, 6-VIII-1984, (1, CCC), Pinus banksiana.

Distribution in Canada. ON, QC, NS.

Pityophthorus carinatus carinatus Bright, 1978

http://species-id.net/wiki/Pityophthorus_carinatus_carinatus

Fig. 9

Record from Bright (1981): Sainte-Anne-du-Lac, Antoine-Labelle, 4-VII-1978, (2, CNC), Pinus strobus.

New records. Lac Needham, Pontiac, 12-VII-2001, (4, MRNQ), Pinus strobus; Saint-Augustin, Maria-Chapdelaine, 28-VIII-1981, (1, MRNQ), Pinus banksiana; Notre-Dame-du-Rosaire, Lac Saint-Jean-Est, 26-V-1981, (3, MRNQ), Picea glauca; Boilleau, Papineau, 29-VII-2004, (1, MRNQ), Picea mariana; Cookshire, Compton, 23-V-2000, (2, LFRC), Pinus resinosa; Johnville, Compton, 24-V-1987, (1, CLC) / 17-V-1987, (1, CLC) / 18-V-1988, (1, CLC) / 21-V-1989 (1, CLC) / 11-VI-1989, (1, CLC); Dosquet, Lotbinière, 16-V-1976, (5, CCC); Lac Cayamant, La-Vallée-de-la-Gatineau,17-VI-2008, (1, INRS-IAF); Fort-Coulonge, Pontiac, 16-VI-2011, (1, INRS-IAF).

Distribution in Canada. QC, NB.

Pityophthorus balsameus Blackman, 1922

http://species-id.net/wiki/Pityophthorus_balsameus

Fig. 7

New records. Cap-Saint-Ignace, Montmagny, 26-VIII-1998, (2, MRNQ), Picea abies / 4-IX-1998, (2, MRNQ), Picea abies; Ways-Mills, Coaticook, 13-V-1988, (5, MRNQ), Pinus strobus; Doncaster Township, Les Laurentides, 7-VII-1981, (1, MRNQ), Pinus resinosa; Saint-Philémon, Bellechasse, 29-V-1981, (1, MRNQ), Pinus resinosa; Armagh, Bellechasse, 16-IX-1979, (1, MRNQ), Pinus resinosa; SainteMarguerite, La Nouvelle-Beauce, 6-VII-1981, (4, MRNQ), Picea abies; Mitchell Township, La-Vallée-de-la-Gatineau, 26-VI-1981, (1, MRNQ), Pinus banksiana; Rivière Petit Saguenay, Le Fjord-du-Saguenay, 25-V-1982, (5, MRNQ), Pinus resinosa; Saint-Ferréol-des-Neiges, La-Côte-de-Beaupré, 3-VII-1990, (1, MRNQ), Pinus resinosa; Saint-Joachim-de-Courval, Drummond, 26-VII-2001, (2, MRNQ), Picea abies; Saint-Germain-de-Grantham, Drummond, 24-VII-1981, (1, MRNQ), Pinus banksiana; Sacré-Cour, La Haute-Côte-Nord, 8-VII-1992, (2, MRNQ), Pinus banksiana; Notre-Dame-du-Rosaire, Lac Saint-Jean-Est, 26-V-1981, (1, MRNQ), Picea glauca; Valcartier, La Jacques-Cartier, 3-VIII-1981, (1, MRNQ), Pinus resinosa; Dosquet, Lotbinière, 9-VI-1970, (1, CCC) / 28-IV-1984, (1, CCC).

Distribution in Canada. NT, ON, QC, NB, NS. 


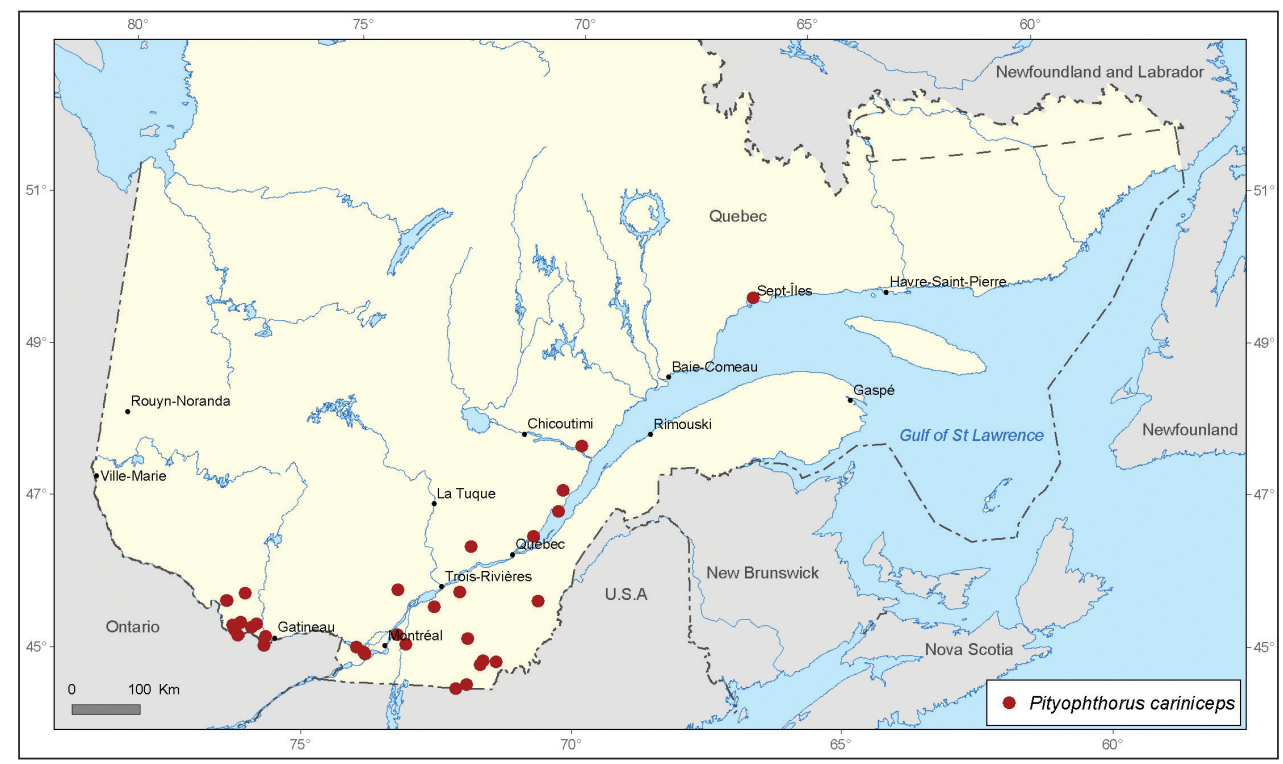

Figure 5. Map of Pityophthorus cariniceps LeConte \& Horn records in Quebec, Canada.

Pityophthorus briscoei Blackman, 1922

http://species-id.net/wiki/Pityophthorus_briscoei

Fig. 6

Record from Bright (1981). Sainte-Anne-du-Lac, Antoine-Labelle, 4-VII-1978, (1, LFRC), Pinus strobus.

New records: Villette, Coaticook, 18-VII-1985, (6, MRNQ), Pinus sylvestris; Saint-Hilarion, Charlevoix, 8-VIII-1990, (1, MRNQ), Picea glauca; Saint-Herménégilde, Coaticook, 8-VII-1985, (3, MRNQ), Pinus resinosa; Poupore, Les Collinesde-l'Outaouais, 30-VI-1992, (4, MRNQ), Pinus banksiana; Simpson Township, Drummond, 19-VIII-1981, (4, MRNQ), Picea glauca; Saint-Joachim-de-Courval, Drummond, 20-VII- 2001, (2, MRNQ), Picea glauca; Island Brook, Le Haut-SaintFrançois, 30-VII-1981, (4, MRNQ), Pinus resinosa; Rawdon Township, Matawinie, 28-VII-1973, (17, MRNQ), Pinus resinosa; Valcartier, La Jacques-Cartier, 23-VII1991, (3, LFRC), Pinus strobus.

Distribution in Canada: ON, QC, NB.

Pityophthorus concavus Blackman, 1928

http://species-id.net/wiki/Pityophthorus_concavus

Fig. 8

Record from Bright (1981). Kazabazua, La Vallée-de-la-Gatineau, 24-VII-1966, (2, CNC), Pinus banksiana. 
New records. Cap-Saint-Ignace, Montmagny, 4-IX-1998, (2, MRNQ), Picea abies / 9.VII.2002, (1, MRNQ), Picea rubens; Armagh, Bellechasse, 16-IX-1979, (1, MRNQ), Pinus resinosa; Doncaster Township, Les Laurentides, 7-VII-1981, (1, MRNQ), Pinus resinosa.

Distribution in Canada. ON, QC, NB, NS.

Pityophthorus ramiperda Swaine, 1917

http://species-id.net/wiki/Pityophthorus_ramiperda

Fig. 9

Records from Bright (1981). L'Île Perrot, Vaudreuil-Soulanges, 30-VII-1910, (1, CNC), Pinus strobus; Sainte-Anne-de-Bellevue, Montréal, 11-VIII-1911, (1, CNC), Pinus strobus.

Record from Paquin and Dupérré (2001). Jamésie, 15-VI-1997, (1, LEMU), Picea mariana.

New records. Paul-Sauvé Park, Deux Montagnes, 27-VII-1982, (4, LFRC), Pinus strobus; Île-du-Grand-Calumet, Pontiac, 31-VIII-1983, (3, LFRC), Pinus strobus.

Distribution in Canada. ON, QC, NS.

Pityophthorus opaculus LeConte, 1878

http://species-id.net/wiki/Pityophthorus_opaculus

Fig. 8

Records from Bright (1981). Sainte-Anne-de-Bellevue, Montréal, ?, (2, CNC); Gaspé Co., 2-VIII-1933, (5, CNC), Picea glauca; Hudson, Vaudreuil-Soulanges, 6-V-1910, (1, CNC), Larix sp.

Records from Paquin and Dupérré (2001). Jamésie, 15-VI-1997, (7, LEMU) and (2, ROUM) Picea mariana / 22-VI-1997, (2, LEMU) and (2, ROUM), Picea mariana I 29-VI-1997, (2, LEMU) and (5, ROUM), Picea mariana / 6-VII-1997, (2, LEMU) and (1, ROUM), Picea mariana / 20-VII-1997, (2, ROUM), Picea mariana / 3-VIII-1997, (1, LEMU), Picea mariana / 10-VIII-1997, (1, ROUM), Picea mariana / 24-VIII-1997, (3, LEMU), Picea mariana / 7-IX-1997, (1, ROUM), Picea mariana / 28-IX-1997, (1, LEMU) and (1, ROUM), Picea mariana; Lac Duparquet, Abitibi, 12-VI-1994, (1, ROUM), Cedrus sp. / 22-VII-1997, (1, ROUM); Lac Labyrinthe, Témiscamingue, 21-VII-1996, (1, ROUM), Abies sp. / 18-VIII-1996, (1, ROUM), Cedrus sp. and Abies sp. stand.

New records. Lac Hubbard, La Vallée-de-la-Gatineau, 31-VII-2001, (3, MRNQ), Picea mariana; Lac Ollivon, La Vallée-de-l'Or, 12-VIII-2002, (3, MRNQ), Picea mariana; Gentilly, Bécancour, 8-VII-2002, (1, MRNQ), Picea glauca; Petite-Rivière-Saint-François, Charlevoix, 19-VIII-1985, (1, MRNQ), Picea abies; Valcartier, La Jacques-Cartier, 13-VIII-1981, (1, MRNQ), Pinus resinosa; Fort-Coulonge, Pontiac, 25-V-2009, (1, INRS-IAF) / 16-VI-2011, (2, INRS-IAF), Pinus strobus / 16-VIII-2011, (2, INRS-IAF), Pinus strobus; Saint-Claude, Le-Val- 


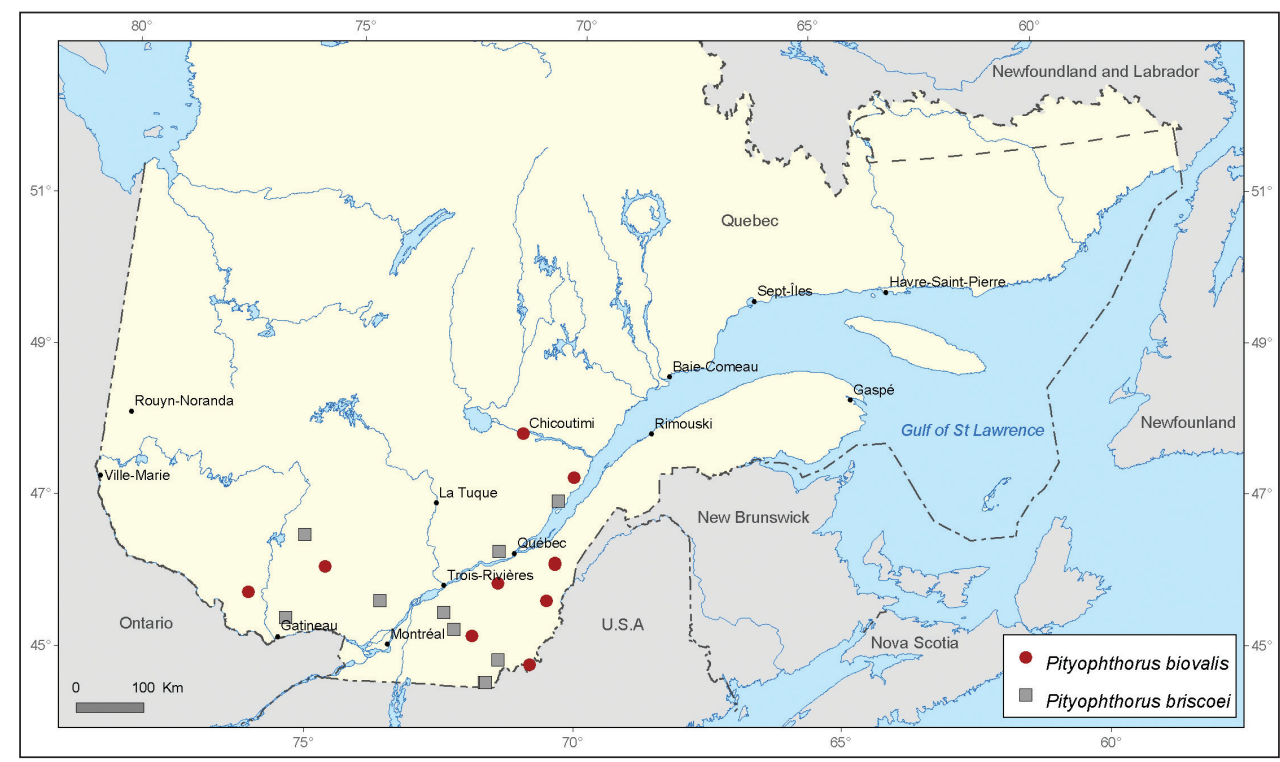

Figure 6. Map of Pityophthorus biovalis Blackman and P. briscoei Blackman records in Quebec, Canada.

Saint-François, 7-VII-2011, (1, INRS-IAF), Pinus strobus; Sept-Îles, Sept-Rivières, 29-V-1987, (1, CCC) / 15-VIII-1987, (1, CCC) / 12-VI-1988, (1, CCC) / 21-VI1988, (1, CCC) / 2-VI-1990, (1, CCC) / 26-VII-1990, (1, CCC) / 23-VIII-1990, (1, CCC) / 6-VIII-1991, (11, CCC) / 9-VIII-1991, (1, CCC) / 13-VIII-1991, (1, CCC).

Distribution in Canada. YK, NT, AB, BC, SK, MB, ON, QC, NB, NS, NF \& LB.

Pityophthorus dentifrons Blackman, 1922

http://species-id.net/wiki/Pityophthorus_dentifrons

Fig. 7

Records from Bright (1981). Gaspé Co., 2-VIII-1933, (10, CNC), Picea glauca.

New records. Sainte-Eulalie, Nicolet-Yamaska, 11-IX-1984, (2, MRNQ), Picea sp., Fort- Coulonge, Pontiac, 25-V-2009, (1, INRS-IAF), Pinus strobus; SaintSimon-les-Mines, Beauce-Sartigan, 30-VI-2009, (1, INRS-IAF), Pinus strobus.

Distribution in Canada. AB, ON, QC, NB, PE, NS, NF \& LB.

Pityophthorus puberulus (LeConte, 1868)

http://species-id.net/wiki/Pityophthorus_puberulus

Fig. 1

Records from Bright (1981). Kazabazua, La Vallée-de-la-Gatineau, 24-VIII1966, (3, CNC), Pinus banksiana; Campbell's Bay, Pontiac, 24-VI-1978, (1, CCC); Sainte-Anne-de-Bellevue, Montréal, ?, 1910, (15, CNC), Pinus sp. / one specimen 
supposedly originating from the same samples was found at LEMU; Sainte-Marie-deBeauce, La Nouvelle-Beauce, ?-VIII-1975, (6, LFRC), Pinus banksiana.

New records. Saint-Théodore, Matawinie, 25-VII-1984, (6, MRNQ), Pinus resinosa; Réservoir Taureau, Matawinie, 10-VII-1981, (2, MRNQ), Pinus resinosa; Baldwin-Mills, Coaticook, 8-VII-1985, (2, MRNQ), Pinus sylvestris; Sainte-Edwidge, Coaticook, 8-VIII-1990, (2, MRNQ), Pinus sylvestris; SaintHerménégilde, Coaticook, 8-VII-1985, (6, MRNQ), Pinus sylvestris; Trois-Lacs, Le Granit, 24-VII-1986, (10, MRNQ), Pinus resinosa / 29-VII-1987, (3, MRNQ), Pinus resinosa; Notre-Dame-des-Bois, Le Granit, 8-VIII-1978, (7, MRNQ), Pinus strobus / 26-VII-1979, (5, MRNQ), Pinus resinosa; Clinton Township, Le Granit, 4-VI-1981, (3, MRNQ), Pinus sylvestris; Chénéville, Papineau, 5-VIII1987, (4, MRNQ), Pinus resinosa; Ripon, Papineau, 8-VII-1992, (1, MRNQ), Pinus banksiana; Notre-Dame-de-la-Paix, Papineau, 16-VII-1993, (2, MRNQ), Pinus resinosa; Lac Quatre Chemins, Papineau, 1-IX-1983, (3, MRNQ), Pinus resinosa; Charteris, Pontiac, 24-V-2000, (2, MRNQ), Pinus sylvestris / 6-VI2000, (2, MRNQ), Pinus banksiana; Thorne, Pontiac, 24-V-2000, (1, MRNQ), Pinus resinosa; Lac Prendergast, Pontiac, 19-VI-1980, (1, MRNQ), Pinus strobus; Lac de la Ferme, Pontiac, 12-VI-1980, (1, MRNQ), Pinus resinosa; Vinton, Pontiac, 31-V-2011, (2, MRNQ), Pinus banksiana; Lac Lacaille, Les Collinesde-l'Outaouais, 30-VIII-1981, (2, MRNQ), Pinus resinosa; Lac Hamilton, Les Collines-de-l'Outaouais, 26-VIII-1983, (2, MRNQ), Pinus resinosa; Lac de la Grande Fourche, Rivière-du-Loup, 6-VIII-1979, (4, MRNQ), Pinus resinosa; Saint-Pierre-de-Lamy, Témiscouata, 25-VII-1985, (4, MRNQ), Pinus resinosa; Cookshire, Le Haut-Saint-François, 11-VIII-1999, (1, MRNQ), Pinus strobus / 23-V-2000, (2, LFRC), Pinus resinosa / 30-V-2000, (1, LFRC), Pinus resinosa / 14-VI-2011, (1, LFRC), Pinus resinosa; Scotstown, Le Haut-Saint-François, 23IX-1999, (1, MRNQ), Pinus sylvestris; Bishopton, Le Haut-Saint-François, 23V-2001, (1, LFRC), Pinus sylvestris / 30-V-2011, (1, LFRC), Pinus resinosa / 24V-2011, (1, LFRC), Pinus resinosa; Waterville, Compton, 17-V-2011, (1, LFRC), Pinus resinosa / 24-V-2011 (3, LFRC), Pinus resinosa / 7-VI-2011, (1, LFRC), Pinus resinosa / 14-VI-2011, (4, LFRC), Pinus resinosa; Johnville, Compton, 14VI-2011, (1, LFRC), Pinus resinosa / 27-V-1987 (1, CLC) / 21-VI-1987 (1, CLC) / 19-VII-1987 (1, CLC) / 11-V-1988 (1, CLC) / 22-V-1988 (1, CLC) / 29-V-1988 (1, CLC) / 1-VI-1988 (1, CLC) / 6-VII-1988 (1, CLC) / 21-V-1989 (1, CLC) / 24-V-1989 (1, CLC) / 25-VI-1989 (2, CLC) / 6-VII-1989 (1, CLC) / 9-VII-1989 (1, CLC) / 16-VII-1989 (1, CLC) / 21-IX-1989 (1, CLC); Huntingville, Sherbrooke, 14-VI-2011, (4, LFRC), Pinus resinosa; Sainte-Marie-de-Blandford, Bécancour, 29-VI-1981, (2, MRNQ), Pinus resinosa; Lemieux, Bécancour, 27-VII1981, (3, MRNQ), Pinus banksiana; Sainte-Séraphine, Arthabaska, 5-VIII-1993, (2, MRNQ), Pinus resinosa; Victoriaville, Arthabaska, 4-X-1978, (10, MRNQ), Pinus resinosa; Saint-Didace, D’Autray, 19-VIII-1978, (1, MRNQ), Pinus resinosa; Berthierville, D’Autray, 4-IX-1997, (2, MRNQ), Pinus resinosa / 27-V1976, (5, CCC), Pinus strobus; Ruisseau Sainte-Émilie, D’Autray, 19-VIII-1979, 


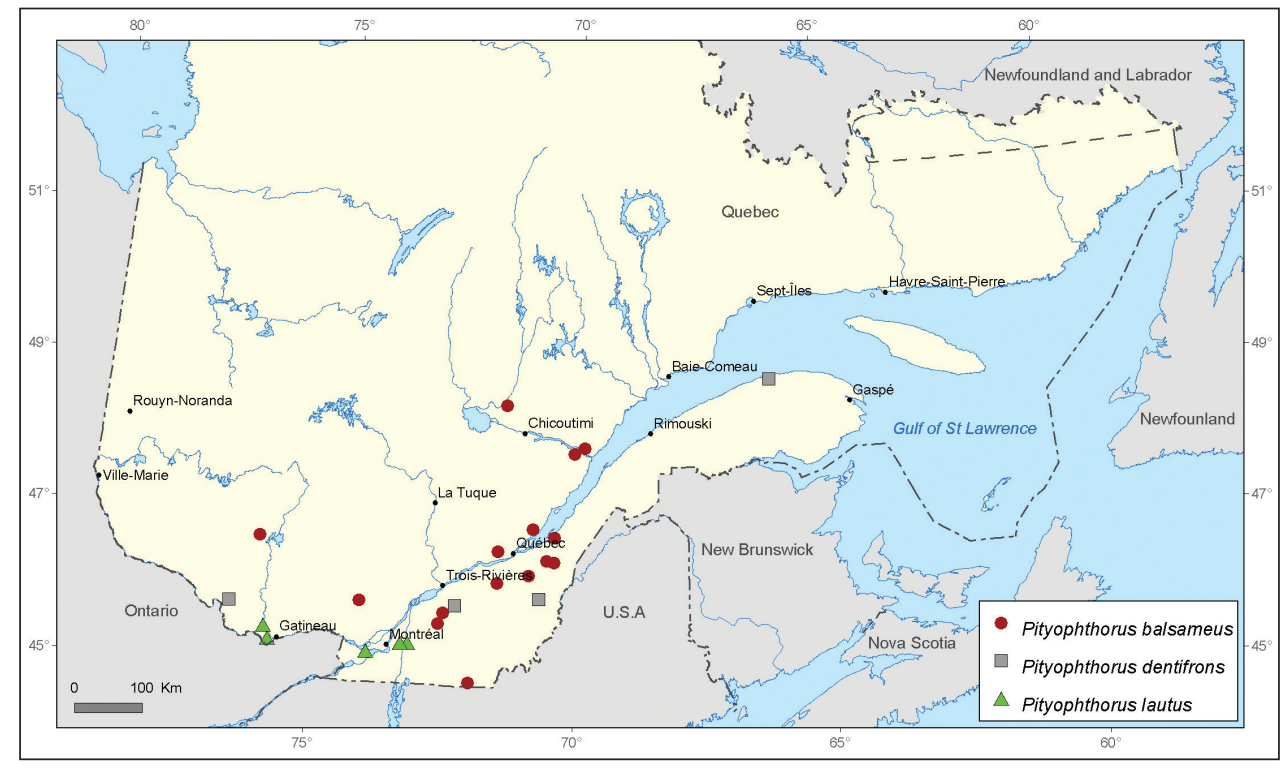

Figure 7. Map of Pityophthorus balsameus Blackman, P. dentifrons Blackman and P. lautus Eichhoff records in Quebec, Canada.

(1, MRNQ), Pinus resinosa; Bromptonville, Le Val-Saint-François, 31-VII-1981, (3, MRNQ), Pinus resinosa; Saint-Adelphe, Mékinac, 30-VII-1986, (5, MRNQ), Pinus resinosa; Lac Éclairé, Mékinac, 30-VII-1980, (2, MRNQ), Pinus resinosa; Saint-Pierre-Montmagny, Montmagny, 7-VIII-1979, (3, MRNQ), Pinus banksiana; Lac Morigeau, Montmagny, 31-VII-2000, (3, MRNQ), Pinus resinosa; SaintDavid-de-Falardeau, Le Fjord-du-Saguenay, 8-VII-2000, (1, MRNQ), Pinus resinosa; Ferland-et-Boilleau, Fjord-du-Saguenay, 18-VII-1988, (1, MRNQ), Pinus banksiana / 11-VII-2011, (1, MRNQ), Pinus banksiana; Mont Saint-Hilaire, La Vallée-du-Richelieu, 4-VI-2011, (1, LFRC); Parc de la Mauricie, Le Centrede-la-Mauricie, 5-VI-2000, (1, LFRC), Pinus strobus; Saint-Basile, Portneuf, 13-VII-2011, (1, MRNQ), Pinus banksiana; Sainte-Luce, La Mitis, 1-VI-1987, (3, MRNQ), Pinus resinosa; Saint-Élie-d'Orford, Sherbrooke, 22-VIII-1978, (3, MRNQ), Pinus resinosa; Saint-Zéphirin-de-Courval, Nicolet-Yamaska, 15-VI1982, (2, MRNQ), Pinus resinosa; Valcartier, La Jacques- Cartier, 3-VIII-1981, (3, MRNQ), Pinus resinosa; Lac Beemer, Le Domaine-du-Roy, 18-VII-2007, (1, MRNQ), Pinus banksiana; Shenley Township, Beauce-Sartigan, 2-VII-1981, (1, MRNQ), Pinus resinosa; Saint-Chrétien, Charlevoix-Est, 31-VIII-1984, (3, MRNQ), Pinus banksiana; Baie-Trinité, Manicouagan, 10-VI-1987, (1, MRNQ), Pinus banksiana / 31-V-1999, (3, MRNQ), Pinus resinosa / 1-VI-1999, (3, MRNQ), Pinus resinosa; L'Annonciation, Antoine-Labelle, 28-VII-1988, (4, MRNQ), Pinus resinosa; Saint-Patrice-de-Beaurivage, Lotbinière, 30-VIII-1989, (6, MRNQ), Pinus resinosa; Landrienne, Abitibi, 15-VII-1987, (1, MRNQ), Pinus banksiana; Saint-Dominique, Abitibi Ouest, 14-VII-2011 (1, MRNQ), 


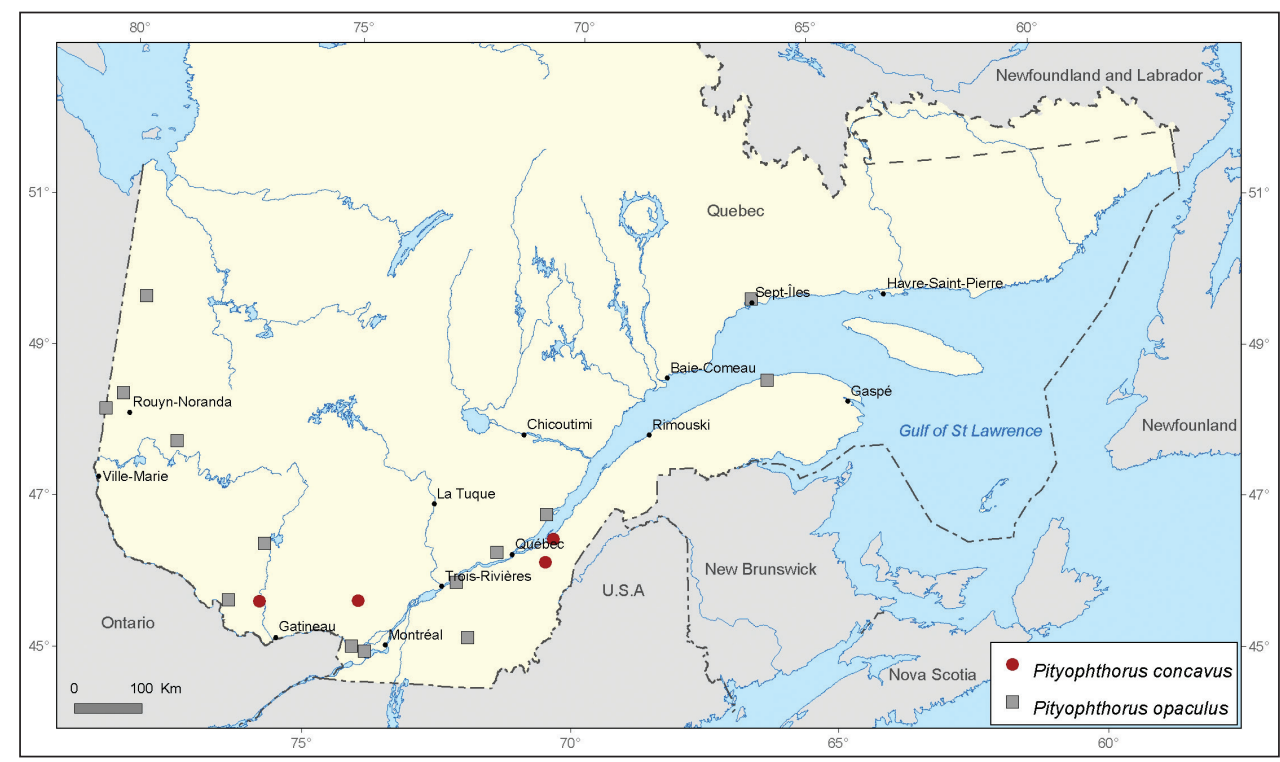

Figure 8. Map of Pityophthorus concavus Blackman and P. opaculus LeConte records in Quebec, Canada.

Pinus banksiana; Lachenaie, Les Moulins, 5-VI-2000, (6, LFRC); Saint-Amable, Marguerite D'Youville, various dates in the period 2008-2012, (12594, INRSIAF), Pinus strobus / 25-V-2000, (2, MRNQ), Pinus strobus; Saint-Claude, Le Val-Saint-François, 31-VII-1981, (9, MRNQ), Pinus sylvestris / 31-V-1985, (18, MRNQ), Pinus resinosa / various dates in the period 2008-2012, (380, INRS-IAF), Pinus strobus; Fort-Coulonge, Pontiac, various dates in the period 2008-2012, (3391, INRS-IAF), Pinus strobus/ 4-VII-2007, (1, MRNQ), Pinus resinosa; Lac Cayamant, La Vallée-de-la-Gatineau, various dates in the period 2008-2012, (38, INRS-IAF), Pinus strobus; Saint-Simon-les-Mines, Beauce-Sartigan, various dates in the period 2008-2012, (564, INRS-IAF), Pinus strobus; Cap-Tourmente, La Côte-de-Beaupré, various dates in the period 2008-2012, (368, INRS-IAF), Pinus strobus; Saint-Étienne, Lévis, 2-VI-1981, (1, CCC) / 10-VI-1983, (1, CCC); Les Escoumins, La Haute-Côte-Nord, 18-VI-1984, (3, CCC).

Distribution in Canada. ON, QC, NB, NS.

Pityophthorus consimilis LeConte, 1878

http://species-id.net/wiki/Pityophthorus_consimilis

Fig. 10

Records from Bright (1981). Sainte-Anne-de-Bellevue, Montréal, ?, (4, CNC); Gaspé Co., 15-VIII-1934, (1, CNC), Picea glauca; Kazabazua, La Vallée-de-la-Gatineau, 13VII-1967, (52, CNC), Pinus banksiana; Sainte-Julienne (Kelly's Camp), Montcalm, 17- VII-1939, (2, CNC), Picea glauca; Lac Mud, Papineau, 24-X-1967, (1, CNC). 


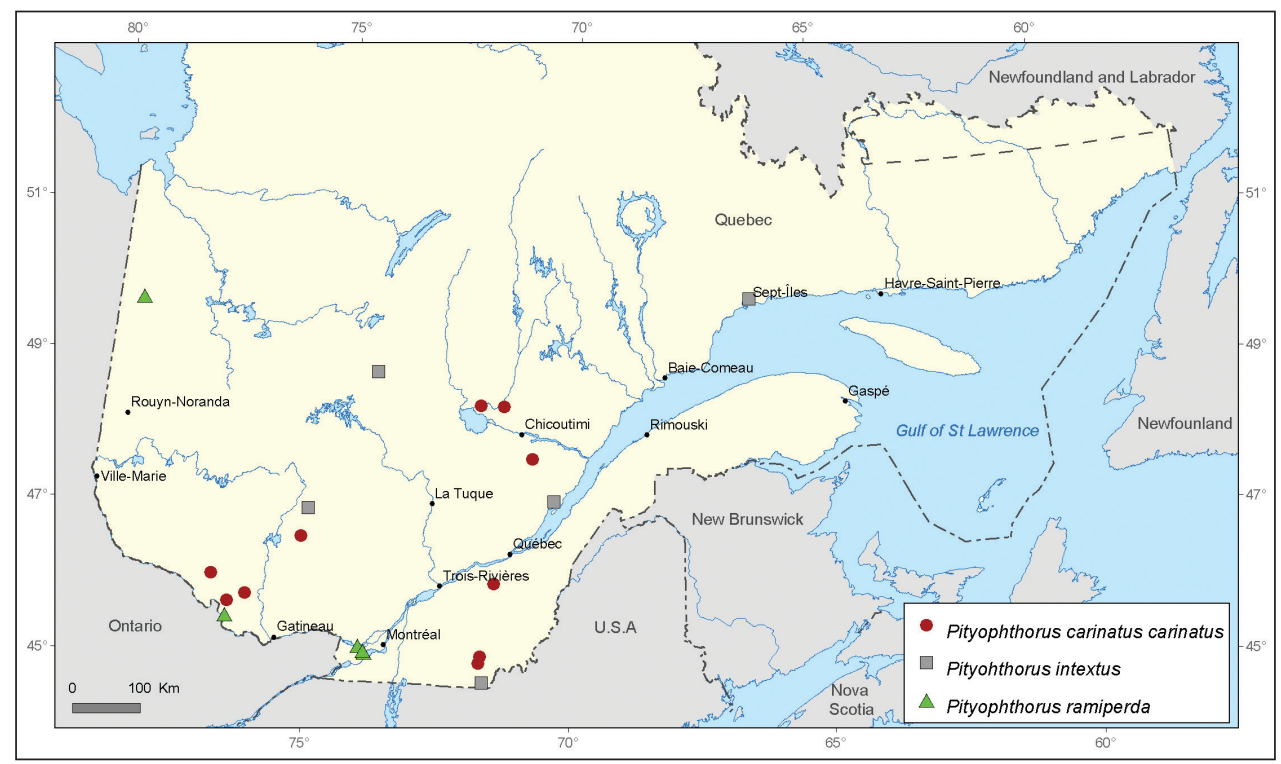

Figure 9. Map of Pityophthorus carinatus carinatus Bright, P. intextus Swaine and P. ramiperda Swaine records in Quebec, Canada.

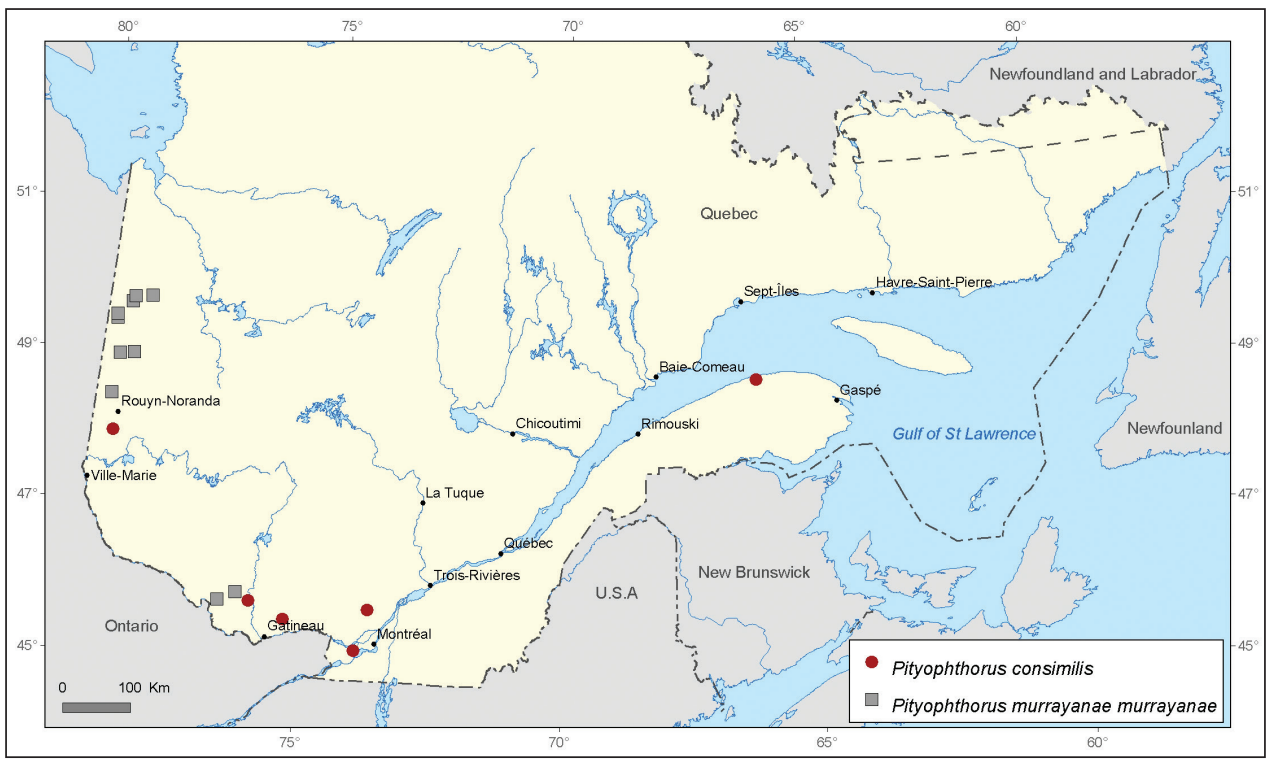

Figure 10. Map of Pityophthorus consimilis LeConte and P. murrayanae murrayanae Blackman records in Quebec, Canada.

New records. Cloutier, Rouyn-Noranda, 30-VII-1981, (1, MRNQ), Pinus banksiana.

Distribution in Canada. AB, BC, MB, SK, ON, QC, NS. 


\section{Pityophthorus murrayanae murrayanae Blackman, 1922}

http://species-id.net/wiki/Pityophthorus_murrayanae_murrayanae

Fig. 10

Records from Paquin and Dupérré (2001). Lac Duparquet, Abitibi, 19-VI-1994, (1, LEMU); Jamésie, 15-VI-1997, (1, LEMU), Picea mariana / 29-VI-1997, (1, LEMU), Picea mariana / 13-VII-1997, (1, LEMU), Picea mariana / 27-VII-1997, (1, LEMU), Picea mariana / 6-VIII-1997, (1, LEMU), Picea mariana / 28-IX-1997, (1, LEMU), Picea mariana.

New records. Fort-Coulonge, Pontiac, various dates in the period 2008-2012, (1005, INRS-IAF), Pinus banksiana; Lac Cayamant, La-Vallée-de-la-Gatineau, various dates in the period 2008-2012, (60, INRS-IAF), Pinus banksiana; Lac Villebois, Jamésie, 14-IX-2006, (1, MRNQ), Pinus banksiana.

Distribution in Canada. NT, AB, BC, MB, ON, QC, NB.

\section{Discussion}

A total of 21690 specimens of Pityophthorus originating from nine entomological collections were analyzed. A total of 291 new localities in Quebec, Canada were recorded for the 17 identified species. The most widespread species in the province of Quebec is P. puberulus with 72 records, followed by P. pulchellus pulchellus with 62 records, then by $P$. pulicarius with 40 records, P. nitidus with 34 records, and P. cariniceps with 33 records.

$P$. puberulus displays a typical northeastern distribution in North America. This twig beetle breeds in various species of Pinus, as well as Abies and Picea (Bright 1981). All $P$. puberulus specimens examined in the province of Quebec were found only on Pinus sp., particularly on Pinus strobus and Pinus resinosa and rarely on Pinus sylvestris and Pinus banksiana. Accordingly, the distribution of P. puberulus in Quebec is positively correlated with the distributions of Pinus strobus and Pinus resinosa. P. puberulus is also a very abundant species, and thousands of specimens may be collected during a field trapping season. Sixty-nine new distribution records are presented for this species in Quebec, Canada. According to Deyrup and Kirkendall (1983), P. puberulus reproduces exclusively parthenogenetically at least in part of their distribution area, which may explain their high abundance. Nevertheless, Bright (1981) described the male of this species.

P. pulchellus pulchellus is one of the most widespread species in North America (Bright 1981). The specimens of this species captured in Quebec were found on different pine species, although mainly on jack pine, Pinus banksiana. It is highly likely that the lack of occurrence in the northern area of Quebec may be determined by the lack of samples above $51^{\circ} \mathrm{N}$. Fifty-eight new locality records are presented for this species in Quebec.

$P$. pulicarius displays a distribution that is predominantly toward the western area of the province. This species was found in thirty-three new localities in Quebec. The specimens were collected principally on Pinus banksiana, as well as Pinus strobus and 
Pinus resinosa. P. pulicarius may be an aggressive species that attacks living twigs of different species of pine (Craighead 1950).

P. nitidus is a species that is widespread in North America throughout the northern coniferous forest (Bright 1981). This species breeds in different species of Pinus and Picea. In Quebec, this species is distributed predominantly along the Saint-Lawrence River through Anticosti Island, which is the easternmost record (Fig. 4). Thirty-one new records are presented for this species in Quebec.

The distribution of $P$. cariniceps is confined to the southern and, specifically, the southeastern areas of Quebec. Two focal regions of occurrence may be detected for this species in Quebec: the first one is located in Gatineau Valley and the second stretches along the Saint-Lawrence River (Fig. 5). Twenty-nine new distribution records are provided for this species in Quebec. Bright (1981) recognized two types of morphological variation of the female frons and male declivity across the North American distribution area: "canadensis form", which is more frequent within the boreal populations, and "cariniceps form", which predominates in the southern populations. The specimens of $P$. cariniceps analyzed from the province of Quebec displayed both previously mentioned forms and an extreme variation of the "cariniceps form" with a strong and sharp elevation of the female frons. $P$. cariniceps, $P$. biovalis, $P$. carinatus carinatus, $P$. balsameus, $P$. briscoei, and $P$. concavus compose the "cariniceps group". All of these species within the "cariniceps group" are identified mainly according to characteristics of the female frons. However, a significant morphological variation of female frons could be detected in each of the species belonging to this taxonomic group. These variations are sometimes so large that they may lead to misidentification. Therefore, a taxonomic revision of the "cariniceps group" through a combination of their morphological and molecular traits is required.

Several species of Pityophthorus occurring in Quebec, Canada display a narrow distribution across the territory. These include $P$. consimilis with 6 records, $P$. ramiperda and $P$. intextus with 5 records, and $P$. dentifrons and $P$. concavus with only 4 records each.

$P$. consimilis is a rare species in Quebec. Only one new provincial record is presented in the western part of Quebec. The species is also rare in the province of Nova Scotia with only one record (Majka et al. 2007a) and it is absent in other Maritime provinces of Canada (Majka et al. 2007a; Majka et al. 2007b).

P. ramiperda is a very rare species with a limited distribution in the eastern part of North America. This species was initially reported in Canada only in Ontario and Quebec by Bright (1981) and was relatively recently found in the province of Nova Scotia (Majka et al. 2007a). Three new locality records are provided in the province of Quebec for this species, which was most recently cited by Paquin and Dupérré (2001) in the Jamésie Regional County Municipality. This record is the northernmost distribution point of P. ramiperda in North America (Fig. 9). This species was previously captured only on white pine, Pinus strobus. However, in the northern part of Quebec territory, the species has been captured in a Picea mariana stand far beyond the northern distribution limit of Pinus strobus in the province. Consequently, it is highly likely that $P$. ramiperda breed in other coniferous species and not just in white pine. This hypothesis remains to be confirmed. 
P. intextus was first mentioned in Quebec by Laplante et al. (1991) and later in a publication of the Ministère des Ressources Naturelles du Québec (MRNQ 2008), but no locality records were provided. In the taxonomic monograph published by Bright (1981), this species was not mentioned as present in Quebec. We present five new localities for P. intextus in Quebec, and these provide the first reliable distribution data for this species. A closely related species, $P$. cascoensis, which is known to be found in the Northwest Territories, Alberta, Ontario, Newfoundland and Labrador has never been reported in Quebec (Bright 1981; Bright and Skidmore 1997, 2002; Wood and Bright 1987, 1992). According to the distribution in Canada, this species should also be found in Quebec.

$P$. dentifrons displays a predominantly southeastern distribution in North America. It is also a species with scarce representation in Quebec. Only three new locality records are provided.

P. concavus has an eastern North American distribution, as reported by Bright (1981). Although Wood and Bright (1992) mention some records from British Columbia, the data do not appear to be reliable because no specimen originating from this Canadian province is inventoried in CNC. This species is rare in Quebec. Three new records are included in this article, and these constitute the only records of this species since its first mention in the province. No other specimen has been recaptured recently.

The distributions of $P$. lautus, $P$. biovalis, and $P$. murrayanae murrayanae display some peculiarities:

$P$. lautus is a species found in mixed and deciduous forests in Quebec and displays a typical southern distribution. The reduced number of locality records in Quebec may be somewhat explained by the placement of the sampling stations, which were located predominantly in coniferous stands. Consequently, the distribution of this species that we report across the province could be underestimated.

$P$. biovalis, similarly to $P$. intextus, was mentioned by Laplante et al. (1991) and in the publication of Ministère des Ressources Naturelles du Québec (MRNQ 2008), but no locality records are provided. Thus, we consider all nine locality records as new for the province of Quebec.

P. murrayanae murrayanae provided a very interesting case. This subspecies was first recorded in Quebec by Paquin and Dupérré (2001) in the Jamésie Regional County Municipality, which is also the northernmost mentioned distribution record in Quebec. Three new locality records are provided in this paper for P. murrayanae murrayanae, and these represent the second report of this subspecies in Quebec. According to its actual provincial distribution, P. murrayanae murrayanae is found only in the northwestern area of Quebec (Fig. 10). Ten specimens from the CNC collection belonging to this subspecies were verified for morphological variation. Specimens from Alberta (3 specimens), British Columbia (3 specimens), Ontario (2 specimens), and New Brunswick (2 specimens) were analyzed. We observed a slight variation in the dimensions of the granules of the elytra declivity. The specimens with west Canadian occurrence display larger granules, whereas the specimens originating from $\mathrm{New}$ Brunswick harbor very small granules. The size of the granules on the declivity may 
also vary among sexes. Another variable morphological character in $P$. murrayanae murrayanae is related to the pubescence of the female frons (Bright 1981). However, this morphological variation occurs within a population (location) and not necessarily between different locations within the full distribution of the species.

The field samples gathered between 2008 and 2012 in the province of Quebec allowed us to identify some interesting specimens (three specimens) that could not be assigned to any known Pityophthorus species. Despite our increased sampling effort, no other similar specimens were recaptured. Further studies will be needed to confirm if novel Pityophthorus species could be described or these are simply morphological anomalies.

\section{Conclusions}

1. More than $30 \%$ (17 species) of the Pityophthorus fauna recorded in Canada is found in Province of Quebec.

2. In general, the our reported distributions of all Pityophthorus species in Quebec may be biased by the locations of our permanent sampling stations, which were positioned exclusively in conifer seed orchards and predominantly along the primary river valleys and major roads. The greatest number of distribution points is concentrated along the Saint-Lawrence River and Gatineau Valley. The actual positions of the permanent sampling stations in Quebec are principally connected with the timber industry. Future sampling campaigns should be organized above $51^{\circ} \mathrm{N}$ to obtain a more realistic overview of the distribution of Pityophthorus in Quebec.

3. Diverse types of forest ecosystems, as well as the north-south temperature gradient may potentially shelter more than 17 Pityophthorus species. Further studies will be needed to increase the knowledge on the fauna and taxonomy of this twig beetle group in Quebec, Canada.

\section{Acknowledgements}

We would like to express our gratitude to Dr. Patrice Bouchard (Canadian National Collection of Insects and Arachnids, Ottawa, Ontario) and to Dr. Hume Douglas (Canadian Food Inspection Agency, Ottawa, Ontario) for their help with the numerous specimens belonging to the CNC. We are also profoundly indebted to Dr. Jan Klimaszewski and George Pelletier (Curators of the René-Martineau Entomological Collection, Natural Resource Canada, Canadian Forest Service), to Ms. Louise Cloutier (Curator of Robert-Ouellet Entomological Collection, Montreal University), and to Dr. Terry Wheeler and Ms. Stéphanie Bouchard (Curators of the Lyman Entomological Collection, McGill University). We thank the passionate entomologists Claude Chantal and Claire Lévesque for providing essential information on the distribution of some Pityophthorus species in Quebec and for kindly furnishing several specimens from their private collections. A special thanks to Dr. Donald E. Bright (C.P. Gillette 
Museum of Arthropod Diversity, Fort Collins, USA), who kindly accepted to confirm the identification of some of the specimens. We also thank the DGPSP, ministère des Ressources Naturelles du Québec for their financial and field work assistance especially to Régis April and René Chouinard. Natural Sciences and Engineering Research Council of Canada (NSERC) through the iFor Research Consortium also provided financial support for this project.

\section{References}

Blackman MW (1928) The genus Pityophthorus Eichh. in North America: A revisional study of the Pityophthorini, with description of two new genera and seventy-one new species. Technical publication no 25. New York state college of forestry at Syracuse University, 183 pp.

Brauner AM, De Groot P (2007) Response of Pityophthorus puberulus (Coleoptera: Curculionidae: Scolytinae) to pheromones and terpenes. The Canadian Entomologist 139: 742-747. doi: $10.4039 / \mathrm{n} 06-101$

Bright DE (1968) Three new species of Pityophthorus from Canada (Coleoptera: Scolytidae).

The Canadian Entomologist 100: 604-608. doi: 10.4039/Ent100604-6

Bright DE (1971) Bark beetles from New Foundland (Coleoptera: Scolytidae). Annales de la Société Entomologique du Québec 16: 124-127.

Bright DE (1976) The bark beetles of Canada and Alaska (Coleoptera: Scolytidae). Publication 1576, Agriculture Canada, Ottawa, Ontario, 241 pp.

Bright DE (1981) Taxonomic monograph of the genus Pityophthorus Eichhoff in North and Central America. Memoirs of the Entomological Society of Canada 118: 1-378. doi: $10.4039 / \mathrm{entm} 113118 \mathrm{fv}$

Bright DE, Skidmore RE (1991) Two new records of Scolytidae (Coleoptera) from Canada. The Coleopterists Bulletin 45: 368-368.

Bright DE, Skidmore RE (1997) A catalog of Scolytidae and Platypodidae (Coleoptera). Supplement 1 (1990-1994). National Research Council Press, Ottawa, Ontario, 368 pp.

Bright DE, Skidmore RE (2002) A catalog of Scolytidae and Platypodidae (Coleoptera). Supplement 2 (1995-1999). National Research Council Press, Ottawa, Ontario, 523 pp.

Bright DE, Skidmore RE, Dunster K (1994) Scolytidae (Coleoptera) associated with dwarf-hackberry, Celtis tenuifolia Nuttall, in Ontario, Canada. The Coleopterists Bulletin 48: 93-94.

Craighead FC (1950) Insect Enemies of Eastern Forests. U.S. Department of Agriculture, Miscellaneous Publication no. 657, 659 pp.

Cranshaw W (2011) Recently recognized range extension of the walnut twig beetle, Pityophthorus juglandis Blackman (Coleoptera: Curculionidae: Scolytinae), in the western United States. The Coleopterists Bulletin 65: 48-49. doi: 10.1649/0010-065X-65.1.48

Dallara PL (1997) Studies on the distribution, interspecific relationships, host range, and chemical ecology of Pityophthorus spp. (Coleoptera: Scolytidae) and selected insectan associates, and their associations with Fusarium subglutinans f.sp. pini in central coastal California Ph.D. dissertation, University of California, Berkeley, California, USA. 
Dallara PL, Seybold SJ, Meyer H, Tolasch T, Wood DL (2000) Semiochemicals from three species of Pityophthorus (Coleoptera: Scolytidae): Identification and field response. The Canadian Entomologist 132: 889-906. doi: 10.4039/Ent132889-6

De Groot P, De Barr GL (2000) Response of cone and twig beetles (Coleoptera: Scolytidae) and a predator (Coleoptera: Cleridae) to pityol, conophthorin and verbenone. The Canadian Entomologist 132: 843-851. doi: 10.4039/Ent132843-6

Deyrup M, Kirkendall LR (1983) Apparent parthenogenesis in Pityophthorus puberulus (Coleoptera: Scolytidae). Annals of the Entomological Society of America 76: 400-402.

Francke W, Pan M-L, König WA, Mori K, Puapoomchareon P, Heuer H, Vité JP (1987) Identification of 'pityol' and 'grandisol' as pheromone components of the bark beetle, Pityophthorus pityographus. Natürwissenschaften 74: 343-345. doi: 10.1007/BF00367933

Furniss RL, Carolin VM (1977) Western Forest Insects. US Department of Agriculture, Miscelaneous Publication no. 1339, Washington, 654 pp.

Hoover K, Wood DL, Storer AJ, Joseph WF, Bros WE (1996) Transmission of the pitch canker fungus, Fusarium subglutinans f.sp. pini, to Monterey pine, Pinus radiata, by coneand twig-infesting beetles. The Canadian Entomologist 128: 981-994. doi: 10.4039/ Ent128981-6

Kolařik M, Freeland E, Utley C, Tisserat N (2011) Geosmithia morbida sp.nov., a new phytopathogenic species living in symbiosis with the walnut twig beetle (Pityophthorus juglandis) on Juglans in USA. Mycologia 103: 325-332. doi: 10.3852/10-124

Laplante S, Bousquet Y, Bélanger P, Chantal C (1991) Liste des espèces de coléoptères du Québec. Fabreries Supplementum 6: 115-117.

LeConte JL, Horn GH (1876) The Rhynchophora of America, North of Mexico. Proceedings of the American Philosophical Society 15: 1-442.

López S, Quero C, Iturrondobeitia JC, Guerrero A, Goldarazena A (2011) Evidence for (E)pityol as an aggregation pheromone of Pityophthorus pubescens (Coleoptera: Curculionidae: Scolytinae). The Canadian Entomologist 143: 447-454. doi: 10.4039/n11-022

MajkaCG,Anderson DM, McCorquodale DB (2007a) Theweevils (Coleoptera:Curculionoidea) of the Maritime Provinces of Canada, II: New records from Nova Scotia and Prince Edward Island and regional zoogeography. The Canadian Entomologist 139: 397-442. doi: $10.4039 / \mathrm{n} 06-021$

Majka CG, Anderson RS, McAlpine DF, Webster RP (2007b) The weevils (Coleoptera: Curculionoidea) of the Maritime Provinces of Canada, I : New records from New Brunswick. The Canadian Entomologist 139: 378-396. doi: 10.4039/n06-008

McNamara J (1991) Family Scolytidae (bark and ambrosia beetles). In: Bousquet Y (Ed) Checklist of beetles of Canada and Alaska. Publications 1861/E, Agriculture Canada, Research Branch, Ottawa, Ontario, 356-365.

Miller DR, Rabaglia RJ (2009) Ethanol and (-)-a-pinene: Attractant kairomones for bark and ambrosia beetles in the Southeastern US. Journal of Chemical Ecology 35: 435-448. doi: 10.1007/s10886-009-9613-9

MRNQ (2008) Liste des coléoptères. Collection d'insectes du Québec. Ressources Naturelles et Faune, Direction de l'environnement et de la protection des forêts, Québec, 31 pp. 
Paquin P, Dupérré N (2001) Beetles of the boreal forest: a faunistic survey carried out in western Quebec. Proceedings of the Entomological Society of Ontario 132: 57-98.

Rappaport NG, Wood DL (1994) Pityophthorus orarius Bright (Coleoptera: Scolytidae) in a Northern California douglas-fir seed orchard: effect of clone, tree vigor, and cone crop on rate of attack. The Canadian Entomologist 126: 1111-1118. doi: 10.4039/Ent1261119-5

Romón P, Iturrondobeitia JC, Gibson K, Lindgren BS, Goldarazena A (2007) Quantitative association of bark beetles with pitch canker fungus and effects of verbenone on their semiochemical communication in monterey pine forests in Northern Spain. Environmental Entomology 36: 743-750. doi: 10.1603/0046-225X(2007)36[743:QAOBBW]2.0.CO;2

Sakamoto JM, Gordon TR, Storer AJ, Wood DL (2007) The role of Pityophthorus spp. as vectors of pitch canker affecting Pinus radiata. The Canadian Entomologist 139: 864-871. doi: $10.4039 / \mathrm{n} 07-022$

Seybold SJ, Coleman TW, Dallara PL, Dart NL, Graves AD, Pederson LA, Spichiger SE (2012) Recent collecting reveals new state records and geographic extremes in the distribution of the walnut twig beetle, Pityophthorus juglandis Blackman (Coleoptera: Scolytidae), in the United States. Pan-Pacific Entomologist 88: 277-280. doi: 10.3956/2012-32.1

Seybold SJ, Haugen D, O’Brien J, Graves AD (2013) Thousand cankers disease USDA Forest Service, Northeastern Area State and Private Forestry Pest Alert, NA-PR-02-10. http:// www.na.fs.fed.us/pubs/detail.cfm?id=5225 [accessed 14 september 2013]

Stevens RE, Lister CK, Linnane JL (1979) Outbreak of a twig beetle, Pityophthorus opaculus LeConte, in Colorado. The Coleopterists Bulletin 33: 268.

Swaine JM (1918) Canadian Bark-Beetles. Part I. Description of new species. Technical Bulletin. Dominion of Canada, Department of Agriculture, Entomological Branch, Ottawa, $143 \mathrm{pp}$.

Wood SL (1978) A reclassification of the subfamilies and tribes of Scolytidae (Coleoptera). Annales de la Société Entomologique de France 14: 95-122.

Wood SL (1982) The bark and ambrosia beetles of North and Central America (Coleoptera: Scolytidae), a taxonomic monograph. Great Basin Naturalist Memoirs 6: 1-1359.

Wood SL (2007) Bark and ambrosia beetles of South America (Coleoptera: Scolytidae) Monte L. Bean Life Science Museum, Brigham Young University, Provo, UT, USA, 900 pp.

Wood SL, Bright DE (1987) A catalog of Scolytidae and Platypodidae (Coleoptera), Part 1: Bibliography. Great Basin Naturalist Memoirs 11: 1-685.

Wood SL, Bright DE (1992) A catalog of Scolytidae and Platypodidae (Coleoptera), Part 2: Taxonomic Index, Volume B. Great Basin Naturalist Memoirs 13: 834-1081. 